\title{
Application of Various Extracts Enhances the Growth and Yield of Cucumber (Cucumis sativus L.) without Compromising the Biochemical Content
}

\author{
Se Ji Jang, Hyun Hwa Park and Yong In Kuk*
}

Citation: Jang, S.J.; Park, H.H.; Kuk, Y.I. Application of Various Extracts Enhances the Growth and Yield of Cucumber (Cucumis satious L.) without Compromising the Biochemical Content. Agronomy 2021, 11, 505. https://doi.org/10.3390/ agronomy11030505

Received: 24 February 2021

Accepted: 4 March 2021

Published: 8 March 2021

Publisher's Note: MDPI stays neutral with regard to jurisdictional claims in published maps and institutional affiliations.

Copyright: (c) 2021 by the authors. Licensee MDPI, Basel, Switzerland. This article is an open access article distributed under the terms and conditions of the Creative Commons Attribution (CC BY) license (https:// creativecommons.org/licenses/by/ $4.0 /)$.
Department of Oriental Medicine Resources, Sunchon National University, Suncheon 57922, Korea; sjsj@scnu.ac.kr (S.J.J.); camellia9720@s.scnu.ac.kr (H.H.P.)

* Correspondence: yikuk@sunchon.ac.kr; Tel.: +82-61-750-3286
Abstract: This study examined the effects of changes to application methods, application time, and combinations of different extracts on the growth and yield of cucumbers. Chlorophyll and photosynthetic efficiency levels in cucumber plants were also measured after receiving treatments of selected liquid fertilizers. Mineral nutrition levels in both extracts themselves and the test plants were measured. Crude protein, crude fat, ash, total phenol and flavonoid contents, and DPPH radical scavenging activity in cucumber were determined. Cucumber plant height and shoot fresh weight at 7 and 14 days after treatments generally increased significantly regardless of the extraction methods or extract materials. On the other hand, cucumber leaf number was similar regardless of the extracts used. The application frequency effects differed according to the extraction methods, extracts, and parameters investigated. In addition, the combinations of extracts did not produce significant increases in cucumber plant growth. Cucumber fruit weight was $17-81 \%, 10-61 \%$, and $10-45 \%$ higher than the control or oil cake when the plants were treated with fermentation, boiled water, and water extracts, respectively. The chlorophyll content and photosynthetic efficiency of cucumber plants treated with various extracts did not vary significantly. Extracts with the highest mineral nutrients were not effective growth promotors of cucumber plants. This means that growth promotion may not be caused by high levels of one specific macro or micro element but by some specific combinations of various elements. Compared to the control, most elements in the cucumber leaves decreased significantly when treated with most of the extracts. Generally, crude protein, crude fat, and ash contents were not negatively affected by the extract treatments. Moreover, most of the extracts did not adversely affect total phenol and flavonoid contents and DPPH radical scavenging activity. Thus, these extracts may be used for growth promotion without negative effects to primary and secondary substances in organically cultivated crop fields.

Keywords: biochemical content; Cucumis sativus; growth promotion; nutrition; photosynthesis; plant extract

\section{Introduction}

As restrictions on the use of chemical fertilizers increase, raw organic materials that are safe for humans and the environment must be used at higher rates to promote crop growth [1]. Therefore, organic agriculture uses crop rotation, allows for fallow fields, and uses green manure cultivation rather than chemical fertilizers for crop cultivation. On the other hand, these methods can cause a nutrient deficiency in organically cultivated crops with longer cultivation periods, such as cucumbers [1,2]. An effective means of combating this problem is the use of liquid fertilizers that can supplement the nutrient needs of crops. Fortunately, such fertilizers can be made using plant by-products, such as soybean oil cake, or waste products, such as onion, tomato, and red pepper leaves [2,3]. These source materials are high in mineral elements, vitamins, amino acids, and oligosaccharides that can improve crop yield and quality [4-6]. On the other hand, producing liquid fertilizers 
with consistent levels of effectiveness is difficult. For example, tomato, cucumber, and red pepper crops experience higher rates of growth promotion when treated with water and boiled water extracts made from tomato, onion, sesame oilcake, and fish powder than when treated with fermentation extracts [3,7]. Another factor that affects the rates of growth promotion is the application method. In one study, liquid fertilizers applied using the drenching method were more effective growth promotors when applied through foliar application $[4,8,9]$. Therefore, careful consideration must be given to the materials, manufacturing methods, and extraction methods used for liquid fertilizer production, as well as the application method, frequency, and concentration $[6,10]$.

Although many liquid fertilizers have been used in horticultural crops for growth promotion, their effectiveness is often quite low when compared to chemical fertilizers [3,4,9]. Additionally, there have been a very limited number of studies on growth promotion of liquid fertilizers with different application methods in cucumber plants. Therefore, this study was carried out to find liquid fertilizers that were quality growth promotors, particularly when applied to cucumber plants.

The mechanisms through which liquid fertilizers improve crop growth are not completely understood. Khan et al. [11] reported that plant growth and development after applying a seaweed extract are related to increased mineral uptake and photosynthetic capacity. In addition, in other studies, chlorophyll content increased following seaweed extract application [12-14]. Plant extracts containing bioactive compounds stimulate the growth and development of vegetable crops with increasing nutrient use efficiency [15]. Moringa oleifera leaves have high nutritional value because they are rich in fiber, protein, carbohydrates, essential amino acids, essential minerals, and vitamins [16]. In addition, the leaves also contain cytokinin, macro, and micro elements [17]. Therefore, farmers can use its leaves as a possible substitute for inorganic fertilizers or as a crop supplement [18]. Therefore, in order to better understand the mechanisms through which liquid fertilizers improve crops, this study examined both the chlorophyll and the plant photosynthetic efficiency as well as mineral content because these have been linked to growth promotion in aforementioned studies.

Plant primary metabolites, such as sugars, amino acids, proteins, nucleic acids, and polysaccharides, are required for plant growth, development, and stress defense [19]. Secondary metabolites, such as phenolic acids and flavonoids, are produced by the plant cell through metabolic pathways derived from the primary metabolic pathways. These secondary metabolites often play an important role in the plant defense against disease. Generally, secondary metabolites, phenolic acids and flavonoids, are produced constitutively in plants during normal growth and development [20]. These compounds can also be induced by treatment with various biotic and abiotic stresses, such as chilling, salt, and drought [21-24]. Moreover, some plant extract treatments can increase the levels of secondary metabolites, such as total phenol and flavonoid contents, which increased significantly after the spinach leaves were treated with an Ascophyllum nodosum extract $(1.0 \mathrm{~g} / \mathrm{L})$ [25]. Similar previous studies have not measured the degree to which primary or secondary substances might be increased in cucumber plants that receive extract applications, so in our study we measured changes to primary and secondary substances to determine whether or not these applications might also increase the overall nutritional quality of crops.

This study assessed various application methods (application time and extract combination treatment) of various liquid fertilizers in terms of their ability to promote growth and yield in cucumbers. Chlorophyll levels and photosynthetic efficiency in cucumber plants were also measured after the treatments with the selected liquid fertilizers. Mineral nutrition levels in both extracts and the test plant were then measured. Finally, crude protein, crude fat, ash, total phenol and flavonoid contents, and DPPH radical scavenging activity in the cucumber were determined after various extract treatments. 


\section{Materials and Methods}

\subsection{Liquid Fertilizers}

The fermentation liquid fertilizers used in this study were made from fish, bone + fish meal, onion, tomato, red pepper leaves, and sesame oil cake. These liquid fertilizers were selected because a previous study reported the highest rates of seedling growth promotion in tomato and red pepper plants [3,6,7]. After these fertilizers were selected, 18 new fertilizers were prepared by altering the material composition, extract volume, and material concentration, as well as using water and boiled water extraction methods [26].

\subsection{Plant Cultivation and Treatment Conditions}

Cucumber seeds (cv. Sinasiaeuncheon) were purchased from Asia Seed Korea Co., Ltd. (Seoul, Korea). They were sown into a 50-cell tray filled with commercial soil (Sunghwa Co., Ltd., Beolgyo, Korea). Ten days after sowing, each germinated seedling was transplanted into its own vinyl pot $(180 \mathrm{~mm})$. Plants were treated with $1 \%$ concentrations of extracts made using either fermentation, boiled water or water extraction methods of fish, bone + fish meal, tomato, onion, red pepper leaf or sesame oil cake. Applications were used to drench the soil 30 days after sowing (3-leaf stage). Applications were made either once or twice. Plants that received two applications received their second application 7 days after the first. The application volume was $2000 \mathrm{~L} / \mathrm{ha}$. Plant height and fresh shoot weight were measured 14 days after treatment in a seedling growth study. In the yield tests, each seedling was transplanted into its own Wagner pot $(1 / 5000 \mathrm{a})$. The plants were treated with extracts at $1 \%$ concentrations four times at 20 day intervals, with the first application made at 10 days after transplanting. Plant height and leaf number were measured at 20 and 40 days after the first treatment. At harvest, fruit length and fruit weight were measured. Greenhouse conditions were, on average, maintained at $35 / 25 \pm 2{ }^{\circ} \mathrm{C}$ day/night temperatures with a $12 \mathrm{~h}$ photoperiod, $70 \%$ relative humidity, and a light intensity of $500 \mu \mathrm{mol} \mathrm{m} \mathrm{m}^{-2} \mathrm{~s}^{-1}$. The plants were watered once or twice daily during the experiment. The experiment was designed in completely a randomized block design with three replications.

\subsection{Photosynthetic Efficacy and Total Chlorophyll Reading (SPAD)}

For the chlorophyll fluorescence and SPAD value measurements, the treatment conditions were the same as those mentioned in Section 2.2. A handheld fluorometer-FluorPen FP100 (Photon Systems Instruments, Brno, Czech Republic) was used for the chlorophyll fluorescence measurements to assess the photosynthetic performance. Liquid fertilizers were applied to the seedlings at the 3-leaf stage. The fifth leaves were selected on each plant at 7 and 14 days after treatment for measurements. Before the measurements $\left(\mathrm{F}_{\mathrm{v}} / \mathrm{F}_{\mathrm{m}}\right)$, the leaves were dark adapted for 15 min to open all the antennae pigments.

The relative chlorophyll concentration was determined using a non-destructive method. The SPAD value of the leaf was determined using a chlorophyll meter SPAD-502, according to the Soil Plant Analysis Department Section, Monolta Camera Co., Osaka, Japan. The SPAD values were also measured in the same seedling and at 7 and 14 days after treatment. When measuring both the chlorophyll fluorescence and relative chlorophyll concentration, the measurements were taken from two different areas of the fifth leaf of each plant.

\subsection{Levels of Mineral Nutrition in Extracts and Treated Leaves}

An analysis of mineral nutrition was determined in both the extracts themselves and the leaves of the plants that received the extract treatments according to the Soil and Plant Analysis Method published by the Rural Development Administration [27]. For mineral nutrition in leaves, the extracts at $1 \%$ concentrations were used to drench the soil 30 days after sowing (3-leaf stage). Mineral nutrition in leaves was measured 14 days after treatment. The nitrogen content was analyzed using an automatic nitrogen analyzer (Buchi Co., Flawil, Switzerland). The effective phosphoric acid content was analyzed using an absorbance photometer (Cintra 40, GBC Scientific Equipment, Ltd., 
Melbourne, Australia), and the levels of $\mathrm{K}, \mathrm{Ca}, \mathrm{Mg}$, and micro elements were analyzed by inductively coupled plasma-atomic emission spectroscopy (ICP-AES, Integra XL Dual, Scientific Equipment, Ltd., Victoria, Australia) [3,6].

\subsection{Crude Protein, Crude Fat, and Ash Contents in Cucumber}

Crude protein, crude fat, and ash were measured in the cucumber fruit that had been treated four times with extracts at a $1 \%$ concentration. After harvesting, the cucumbers were dried in a dry oven at $40{ }^{\circ} \mathrm{C}$ for four days. The cucumber was then ground into a fine powder using a grinder (SHMF-3500TG, Seoul, South Korea).

Crude protein content was measured using an automatic nitrogen analyzer (K-446/B324, Büchi, Flawil, Switzerland), to which the micro Kjeldahl method was applied. Then the nitrogen content was calculated from the nitrogen coefficient [28]. The crude fat content was measured using a soxhlet extractor (B-811, Büchi, Flawil, Switzerland) after lipid extraction by petroleum ether as a solvent [28].

Regarding crude ash content, samples were measured out into a clean crucible and weighed before being transferred to the ash furnace (F-48000, Thermolyne, Waltham, MA, USA), where they were heated at $550{ }^{\circ} \mathrm{C}$ for more than $15 \mathrm{~h}$ until white or grayish-white ash was obtained [28]. After cooling, the sample was weighed when it reached room temperature, and the amount of ash of the sample was calculated.

\subsection{Phenol and Flavonoid Contents and DPPH Radical Scavenging Activity}

Total phenol and flavonoid contents and DPPH radical scavenging activity were measured in the cucumber fruit that received the extracts at $1 \%$ concentration four times from seedling to harvest. After harvest, the cucumbers were dried in a dry oven at $40{ }^{\circ} \mathrm{C}$ for four days. The cucumbers were then ground using a grinder (SHMF-3500TG, Seoul, South Korea). To determine total phenol and flavonoid concentrations as well as DPPH radical scavenging activity, $0.2 \mathrm{~g}$ of dried cucumber was mixed with $5 \mathrm{~mL}$ of $100 \%$ methanol. After this initial procedure, the supernatants were used for further analysis [29-31].

In measuring the phenol content, $1 \mathrm{~mL}$ of the supernatant was taken and mixed with $3 \mathrm{~mL}$ distilled water and $1 \mathrm{~mL}$ of Folin-Dennis' reagent and shaken for five minutes. The mixed solution was then left for one hour at room temperature and measured calorimetrically using a UV spectrophotometer at 640 nm (UV1601; Shimadzu Co., Kyoto, Japan) [29-31].

In measuring the flavonoid content, $0.5 \mathrm{~mL}$ of the supernatant was mixed with $1.5 \mathrm{~mL}$ of ethanol (95\%), 10\% $\mathrm{AlCl}_{3}, 1 \mathrm{M}$ potassium acetate, and $2.8 \mathrm{~mL}$ distilled water. The mixed solution was then left for $40 \mathrm{~min}$ at room temperature and measured calorimetrically using a UV spectrophotometer at $415 \mathrm{~nm}$ (UV-1601; Shimadzu Co., Kyoto, Japan) [29-31].

When measuring DPPH radical scavenging activity, $0.1 \mathrm{~mL}$ of supernatant, $0.5 \mathrm{~mL}$ of $0.1 \mathrm{M}$ acetate buffer solution ( $\mathrm{pH} 5.5$ ), $0.25 \mathrm{~mL}$ of $0.5 \mathrm{mM}$ DPPH (2,2-diphenyl-1picrylhydarzyl), and $0.4 \mathrm{~mL}$ ethanol were mixed for $30 \mathrm{~min}$ at room temperature. The DPPH radical scavenging activity of the mixed solution was determined calorimetrically using a UV spectrophotometer at 517 nm (UV-1601; Shimadzu Co., Kyoto, Japan) [29-31].

\subsection{Statistical Analysis}

All experiments except that in Section 2.5 were carried out with 3 replications. The experiment "Crude Protein, Crude Fat, and Ash Contents in Cucumber" in Section 2.5 was carried out only once because all previous experiments' resulting values showed very low variation. Some data are expressed as a percentage of the untreated control for ease of comparison between treatments. The data were analyzed using the analysis of variance (ANOVA) procedure in the Statistical Analysis Systems [32] software. The means were separated using Duncan's Multiple Range Test $(p=0.05)$. 


\section{Results and Discussion}

\subsection{Effect of Various Liquid Fertilizers on Seedling Growth in Cucumbers}

For this study, the liquid fertilizers selected in a previous study were used because they had the highest growth promotion rates $[3,6,7,26,31]$. The selected liquid fertilizers were made from fish, bones + fish, tomato, onion, red pepper leaf, and sesame oil cake with different fermentation, water, and boiled water extraction methods, as shown in Table 1. The extract $\mathrm{pH}$ and EC values were checked for stability. The extracts had nearly constant $\mathrm{pH}$ and EC values during the experimental period (data not shown). The application timing (20 and 40 days after sowing) did not significantly affect the rates of cucumber growth promotion in plants that received the extracts. Therefore, seedlings 30 days after sowing were used for this study.

Seven days after the extract treatments, compared to control, plant height increased significantly when treated with all fermentation and boiled water extracts (Table 1). On the other hand, plant height increased significantly only when the plants received applications of extracts made from fish, bone + fish, and sesame oil cake extracts. Cucumber plant height was greater when the plants received two applications of fermentation tomato and red pepper leaf extracts and boiled water fish, tomato, onion, red pepper leaf, and sesame oil cake extracts rather than one application of those extracts. On the other hand, applications of fermentation bone + fish and sesame oil cake extracts and water sesame oil cake extracts increased plant height at the greatest rates when applied just once. Overall, plant height was similar regardless of whether the plants had been given the applications once or twice. On the other hand, plant height increased at the highest rates when the plants received fermentation and boiled water extract applications instead of the water extracts. Compared to the control, cucumber leaf number at 7 days after treatment was similar regardless of which extract had been applied.

Compared to the control, plants that had received treatments, regardless of the extraction methods or extract materials, had a greater plant height at 14 days after the treatments. Similar to plant height 7 days after treatment, plant height at 14 days after the treatment also increased significantly when some extracts were applied twice and when other extracts were applied just once. On the other hand, plant height generally increased significantly regardless of the application frequency. Compared to the control, cucumber leaf number at 14 days after the treatments was not significantly different regardless of which extracts had been used.

Shoot fresh weight increased significantly in most extracts compared to the control. Shoot fresh weight was greater when the plants received two applications of fermented fish, bone + fish, tomato, and onion extracts than one application of those extracts. On the other hand, one application of boiled water bone + fish, tomato, and onion extracts produced plants with a higher shoot fresh weight than two applications. The application frequency did not significantly affect the shoot fresh weight of plants treated with water extracts. Overall, cucumber shoot fresh weight was greater when the plants received two applications rather than one. This is consistent with a previous study, in which red pepper shoot fresh weight was higher when the plants received two applications of tomato and onion water extracts and tomato boiled water extracts [7]. Nevertheless, the effects of a particular application frequency vary according to the crop to which they are applied, the extraction methods used in manufacturing, the type of extract being applied, between measuring parameters (plant height and shoot fresh weight), and between investigation time at 7 or 14 days after treatment. The results of this study were not consistent between measuring parameters and between investigation time. Similar to this study, in a previous study, although cucumber plant height and shoot fresh weight increased in response to the applications of various extracts at different concentrations and with different application methods, the results of plant height and shoot fresh weight were inconsistent [26]. Shoot fresh weight is a more important parameter than plant height in determining an extract's effectiveness in crop growth promotion. In fact, higher plant height (overgrowth) may have a negative effect on growth promotion. Thus, in the conclusion of this study, we 
focused on shoot fresh weight when determining which extracts were the most effective growth promotors.

Table 1. Application frequency effects on cucumber plant height, leaf number, and shoot fresh weight using drenching applications at $1 \%$ concentrations of various extracts.

\begin{tabular}{|c|c|c|c|c|c|c|c|}
\hline \multirow{2}{*}{$\begin{array}{l}\text { Extraction } \\
\text { Methods }\end{array}$} & \multirow{2}{*}{ Extract } & \multirow{2}{*}{$\begin{array}{l}\text { Application } \\
\text { Frequency }\end{array}$} & \multicolumn{2}{|c|}{7 DAT } & \multicolumn{3}{|c|}{14 DAT } \\
\hline & & & Plant Height & $\begin{array}{c}\text { Leaf } \\
\text { Number }\end{array}$ & Plant Height & $\begin{array}{c}\text { Leaf } \\
\text { Number }\end{array}$ & $\begin{array}{c}\text { Shoot Fresh } \\
\text { Weight }\end{array}$ \\
\hline & & & & 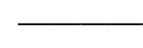 & -- \% of Control & - & \\
\hline \multirow{13}{*}{ Fermentation } & \multicolumn{2}{|c|}{ Control } & 0.0 pqrs* & $0.0^{a b c}$ & $0.0^{\mathrm{s}}$ & $0.0^{b c d}$ & $0.0^{\text {no }}$ \\
\hline & \multirow{2}{*}{ Fish } & Once & $19.7^{\mathrm{ijklm}}$ & $2.9^{a b c}$ & $16.0 \mathrm{ijklmn}$ & $-1.0^{\mathrm{cd}}$ & $4.6^{\mathrm{klmno}}$ \\
\hline & & Twice & $31.4^{\text {defghi }}$ & $-2.0^{b c}$ & 18.1 hijkl & $2.9 \mathrm{bcd}$ & $29.6^{\mathrm{abcd}}$ \\
\hline & \multirow{2}{*}{$\begin{array}{c}\text { Bone }+ \text { fish } \\
\text { meal }\end{array}$} & Once & $54.1^{\mathrm{bc}}$ & $2.9 \mathrm{abc}$ & $19.6^{\text {fghijk }}$ & $2.9 \mathrm{bcd}$ & $12.5^{\text {hijklm }}$ \\
\hline & & Twice & $33.7^{\text {defgh }}$ & $-2.0 \mathrm{bc}$ & $41.5^{\mathrm{ab}}$ & $10.5^{a b}$ & $38.8^{\mathrm{a}}$ \\
\hline & \multirow[b]{2}{*}{ Tomato } & Once & $10.7^{\text {lmnop }}$ & $2.9 \mathrm{abc}$ & $29.0^{c d}$ & $6.7^{\mathrm{abc}}$ & $21.2^{\text {cdefghi }}$ \\
\hline & & Twice & 42.0 cde & $7.8^{a b}$ & $11.4^{\text {lmnop }}$ & $6.7^{a b c}$ & $40.0^{\mathrm{a}}$ \\
\hline & \multirow{2}{*}{ Onion } & Once & 3.9 nopqrs & $-2.0 \mathrm{bc}$ & 18.7 ghijkl & $6.7^{a b c}$ & $9.6^{\mathrm{ijk} k \mathrm{lmn}}$ \\
\hline & & Twice & $35.6^{\text {defg }}$ & $-2.0 \mathrm{bc}$ & 9.9 nopq & $2.9 \mathrm{bcd}$ & $31.3^{\mathrm{abc}}$ \\
\hline & \multirow{2}{*}{$\begin{array}{l}\text { Red pepper } \\
\text { leaf }\end{array}$} & Once & $16.8^{\mathrm{jklmn}}$ & $-2.0^{b c}$ & 21.1 efghi & $10.5^{\mathrm{ab}}$ & $30.3^{a b c}$ \\
\hline & & Twice & $42.3^{\text {cde }}$ & $2.9 \mathrm{abc}$ & $15.9^{\mathrm{ijklmn}}$ & $2.9 \mathrm{bcd}$ & $16.5^{\text {efghijkl }}$ \\
\hline & \multirow{2}{*}{$\begin{array}{l}\text { Sesame oil } \\
\text { cake }\end{array}$} & Once & $41.7^{\text {cde }}$ & $2.9 \mathrm{abc}$ & $13.1^{\mathrm{klmno}}$ & $2.9 \mathrm{bcd}$ & 14.0 fghijklm \\
\hline & & Twice & $11.4^{\text {lmnop }}$ & $2.9^{a b c}$ & $29.4^{\mathrm{cd}}$ & $2.9^{b c d}$ & $25.5^{\text {bcdefg }}$ \\
\hline \multirow{12}{*}{ Boiled water } & \multirow[b]{2}{*}{ Fish } & Once & $-9.7^{\mathrm{s}}$ & $2.9^{a b c}$ & $0.0^{\mathrm{s}}$ & $0.0 \mathrm{bcd}$ & $0.0^{\text {no }}$ \\
\hline & & Twice & 15.0 klmno & $-2.0^{b c}$ & $16.0^{\mathrm{ijklmn}}$ & $-1.0^{\mathrm{cd}}$ & $4.6^{\mathrm{klmno}}$ \\
\hline & \multirow{2}{*}{$\begin{array}{c}\text { Bone + fish } \\
\text { meal }\end{array}$} & Once & $9.3^{1 \mathrm{mnopq}}$ & $2.9^{\mathrm{abc}}$ & $18.1^{\mathrm{hijkl}}$ & $2.9 \mathrm{bcd}$ & $29.6^{\mathrm{abcd}}$ \\
\hline & & Twice & 22.8 ghijkl & $-6.9^{c}$ & $19.6^{\text {fghijk }}$ & $2.9 \mathrm{bcd}$ & $12.5^{\text {hijklm }}$ \\
\hline & \multirow{2}{*}{ Tomato } & Once & $26.1^{\text {fghij }}$ & $2.9^{a b c}$ & $41.5^{\mathrm{ab}}$ & $10.5^{\mathrm{ab}}$ & $38.8^{\mathrm{a}}$ \\
\hline & & Twice & $44.2^{\mathrm{cd}}$ & $2.9 \mathrm{abc}$ & $29.0^{\mathrm{cd}}$ & $6.7^{a b c}$ & $21.2^{\text {cdefghi }}$ \\
\hline & \multirow{2}{*}{ Onion } & Once & 20.9 hijklm & $2.9 \mathrm{abc}$ & $11.4^{\text {lmnop }}$ & $6.7^{a b c}$ & $40.0^{\mathrm{a}}$ \\
\hline & & Twice & $71.3^{a}$ & $12.7^{\mathrm{a}}$ & 18.7 ghijkl & $6.7^{a b c}$ & $9.6^{\mathrm{ijklmn}}$ \\
\hline & \multirow{2}{*}{$\begin{array}{l}\text { Red pepper } \\
\text { leaf }\end{array}$} & Once & 38.9 def & $2.9 \mathrm{abc}$ & 9.9 nopq & $2.9 \mathrm{bcd}$ & $31.3^{a b c}$ \\
\hline & & Twice & $66.9^{a b}$ & $7.8^{a b}$ & 21.1 efghi & $10.5^{a b}$ & $30.3^{a b c}$ \\
\hline & \multirow{2}{*}{$\begin{array}{l}\text { Sesame oil } \\
\text { cake }\end{array}$} & Once & 22.0 ghijkl & $2.9 \mathrm{abc}$ & 15.9 ijklmn & $2.9 \mathrm{bcd}$ & $16.5^{\text {efghijkl }}$ \\
\hline & & Twice & $64.2^{\mathrm{ab}}$ & $2.9^{a b c}$ & 13.1 klmno & $2.9 \mathrm{bcd}$ & $14.0^{\text {fghijklm }}$ \\
\hline \multirow{12}{*}{ Water } & \multirow[b]{2}{*}{ Fish } & Once & $62.5^{a b}$ & $2.9^{a b c}$ & $29.4^{\mathrm{cd}}$ & $2.9 \mathrm{bcd}$ & $25.5^{\text {bcdefg }}$ \\
\hline & & Twice & $54.9^{\mathrm{bc}}$ & $-2.0 \mathrm{bc}$ & $15.5^{\mathrm{ijk} k \mathrm{mn}}$ & $2.9 \mathrm{bcd}$ & $22.2^{\text {cdefgh }}$ \\
\hline & \multirow{2}{*}{$\begin{array}{l}\text { Bone }+ \text { fish } \\
\text { meal }\end{array}$} & Once & 28.9 efghij & $-2.0^{b c}$ & 7.6 opqr & $6.7^{\mathrm{abc}}$ & $7.2^{\mathrm{jklmno}}$ \\
\hline & & Twice & $22.8^{\text {ghijkl }}$ & $2.9 \mathrm{abc}$ & $8.1^{\text {opqr }}$ & $-1.0^{\mathrm{cd}}$ & 13.9 fghijklm \\
\hline & \multirow{2}{*}{ Tomato } & Once & $-3.6^{\mathrm{qrs}}$ & $2.9^{a b c}$ & $17.4^{\mathrm{ijklm}}$ & $-1.0^{\mathrm{cd}}$ & $-3.5^{\mathrm{o}}$ \\
\hline & & Twice & $8.1^{\mathrm{mnopq}}$ & $2.9 \mathrm{abc}$ & $20.8^{\text {efghij }}$ & $2.9 \mathrm{bcd}$ & $16.9^{\text {efghijk }}$ \\
\hline & \multirow{2}{*}{ Onion } & Once & 2.5 opqrs & $-2.0^{b c}$ & 27.9 de & $-1.0^{\mathrm{cd}}$ & $20.4^{\text {cdefghi }}$ \\
\hline & & Twice & $-6.5^{\mathrm{rs}}$ & $-2.0 \mathrm{bc}$ & $35.3 \mathrm{bc}$ & $14.3^{\mathrm{a}}$ & $26.0^{b c d e f}$ \\
\hline & Red pepper & Once & $1.5^{\text {opqrs }}$ & $2.9 \mathrm{abc}$ & $24.9^{\text {defgh }}$ & $2.9 \mathrm{bcd}$ & $3.9 \mathrm{mno}$ \\
\hline & leaf & Twice & -3.5 qrs & $2.9 \mathrm{abc}$ & 25.7 defg & $10.5^{\mathrm{ab}}$ & $15.8^{\text {efghijklm }}$ \\
\hline & Sesame oil & Once & 23.1 ghijkl & $-2.0 \mathrm{bc}$ & $27.2^{\mathrm{de}}$ & $2.9 \mathrm{bcd}$ & 17.7 defghij \\
\hline & cake & Twice & $5.6^{\text {nopqr }}$ & $2.9 \mathrm{abc}$ & 18.9 ghijk & $14.3^{\mathrm{a}}$ & $21.9^{\mathrm{abcd}}$ \\
\hline
\end{tabular}

DAT, days after treatment. * Means within a column followed by the same letters are not significantly different at the $5 \%$ level according to Duncan's Multiple Range Test. 
Although several studies used extracts made from a combination of source materials $[2,3,7,26,30,31,33]$, the present study is the first to examine the effects of combined extracts made from both single or multiple materials. In a previous study, the foliar application of a combination of moringa leaf extract and other growth-promoting substances improved the biochemical traits and growth attributes of wheat [33]. This study aimed to find different combinations of extracts that might also have greater rates of growth promotion than those observed in previous studies (Table 2). At 7 days after treatments, plant height increased significantly after being treated with tomato + sesame oil cake, and onion + sesame oil cake fermentation extract combinations; tomato + sesame oil cake boiled water extract combinations; and tomato + sesame oil cake water extract combinations, compared to the control. Cucumber leaf number increased significantly after being treated with onion + sesame oil cake boiled water extract combinations; and tomato + sesame oil cake, and onion + sesame oil cake water extract combinations, compared to the control. On the other hand, these combinations of extracts did not significantly increase plant height or leaf number than the cucumber plants treated with single material extracts.

Table 2. Effects on cucumber plant height, leaf number, and shoot fresh weight using drenching applications at $1 \%$ concentrations of combined extracts.

\begin{tabular}{|c|c|c|c|c|c|c|}
\hline \multirow{2}{*}{$\begin{array}{l}\text { Extraction } \\
\text { Method }\end{array}$} & \multirow[b]{2}{*}{ Extract } & \multicolumn{2}{|c|}{7 DAT } & \multicolumn{3}{|c|}{14 DAT } \\
\hline & & Plant Height & Leaf Number & Plant Height & Leaf Number & $\begin{array}{c}\text { Shoot Fresh } \\
\text { Weight }\end{array}$ \\
\hline & & \multicolumn{5}{|c|}{$\%$ of Control -} \\
\hline & Control & $0.0^{\mathrm{d} *}$ & $0.0^{\mathrm{b}}$ & $0.0^{\mathrm{e}}$ & $0.0^{\mathrm{c}}$ & $0.0^{\mathrm{e}}$ \\
\hline \multirow{8}{*}{ Fermentation } & Tomato & $30.7^{a b c}$ & $17.2^{\mathrm{a}}$ & $29.8^{a}$ & $6.3^{\mathrm{abc}}$ & $29.4^{\mathrm{abcd}}$ \\
\hline & Onion & $20.1^{\mathrm{abcd}}$ & $2.6^{\mathrm{ab}}$ & $14.6^{\text {abcde }}$ & $3.4^{\mathrm{abc}}$ & $32.7^{\mathrm{abcd}}$ \\
\hline & Fish & $24.8^{\mathrm{abcd}}$ & $13.6^{\mathrm{ab}}$ & $16.8^{\text {abcde }}$ & $6.3^{\mathrm{abc}}$ & $25.9 \mathrm{bcd}$ \\
\hline & Sesame oil cake & $44.6^{\mathrm{a}}$ & $17.2^{\mathrm{a}}$ & $21.7 \mathrm{abcd}$ & $6.3^{\mathrm{abc}}$ & $24.6^{\mathrm{cd}}$ \\
\hline & Tomato + fish & $27.4^{\mathrm{abcd}}$ & $6.2^{\mathrm{ab}}$ & $21.7^{\mathrm{abcd}}$ & $3.4^{\mathrm{abc}}$ & $23.4^{\mathrm{cd}}$ \\
\hline & $\begin{array}{c}\text { Tomato + } \\
\text { sesame oil cake }\end{array}$ & $30.0^{a b c}$ & $13.6^{\mathrm{ab}}$ & $12.3^{\text {abcde }}$ & $6.3^{\mathrm{abc}}$ & $10.8^{\text {de }}$ \\
\hline & Onion + fish & $26.1^{\mathrm{abcd}}$ & $13.6^{\mathrm{ab}}$ & $7.8^{\text {bcde }}$ & $9.2^{a b c}$ & $15.3^{\text {cde }}$ \\
\hline & $\begin{array}{l}\text { Onion + sesame } \\
\text { oil cake }\end{array}$ & $33.3^{\mathrm{ab}}$ & $13.6^{\mathrm{ab}}$ & $9.2^{\text {bcde }}$ & $7.8^{\mathrm{abc}}$ & $20.3^{\text {cde }}$ \\
\hline \multirow{8}{*}{ Boiled water } & Tomato & $13.5^{\mathrm{bcd}}$ & $9.9^{\mathrm{ab}}$ & $4.4^{\text {de }}$ & $3.4^{\mathrm{abc}}$ & $11.1^{\text {de }}$ \\
\hline & Onion & $22.1^{\mathrm{abcd}}$ & $9.9^{a b}$ & $6.8^{\text {cde }}$ & $-2.3^{c}$ & $12.6^{\text {cde }}$ \\
\hline & Fish & $33.3^{a b}$ & $6.2^{a b}$ & 10.0 bcde & $3.4^{\mathrm{abc}}$ & $14.0^{\text {cde }}$ \\
\hline & Sesame oil cake & $36.6^{\mathrm{ab}}$ & $13.6^{\mathrm{ab}}$ & $26.9^{a b}$ & $14.9^{a b c}$ & $46.4^{\mathrm{ab}}$ \\
\hline & Tomato + fish & $12.2^{\mathrm{abcd}}$ & $6.2^{\mathrm{ab}}$ & $8.7^{\text {bcde }}$ & $3.4^{\mathrm{abc}}$ & $20.7^{\text {cde }}$ \\
\hline & $\begin{array}{c}\text { Tomato }+ \\
\text { sesame oil cake }\end{array}$ & $39.9^{a b}$ & $13.6^{\mathrm{ab}}$ & $22.0^{\mathrm{abcd}}$ & $12.1^{\mathrm{abc}}$ & $28.5^{a b c d}$ \\
\hline & Onion + fish & $14.2^{\mathrm{abcd}}$ & $4.4^{\mathrm{ab}}$ & $6.8^{\text {cde }}$ & $3.4^{\mathrm{abc}}$ & $22.4^{\text {cde }}$ \\
\hline & $\begin{array}{l}\text { Onion + sesame } \\
\text { oil cake }\end{array}$ & $28.1^{\mathrm{abcd}}$ & $17.2^{\mathrm{a}}$ & $8.4^{\text {bcde }}$ & $6.3^{a b c}$ & $19.2^{\text {cde }}$ \\
\hline \multirow{8}{*}{ Water } & Tomato & $34.0^{\mathrm{ab}}$ & $9.9^{a b}$ & $6.5^{\text {cde }}$ & $17.8^{\mathrm{ab}}$ & $31.8^{\mathrm{abcd}}$ \\
\hline & Onion & $10.9 \mathrm{bcd}$ & $6.2^{\mathrm{ab}}$ & $1.3^{\mathrm{e}}$ & $6.3^{a b c}$ & $16.8^{\text {cde }}$ \\
\hline & Fish & $3.6^{\mathrm{cd}}$ & $6.2^{\mathrm{ab}}$ & $1.0^{\mathrm{e}}$ & $-2.3^{c}$ & 9.9 de \\
\hline & Sesame oil cake & $18.2^{\mathrm{abcd}}$ & $9.9^{a b}$ & $12.6^{\text {abcde }}$ & $9.2^{\mathrm{abc}}$ & $15.8^{\text {cde }}$ \\
\hline & Tomato + fish & $12.2^{b c d}$ & $9.9^{a b}$ & 9.1 bcde & $0.6^{\mathrm{bc}}$ & $20.4^{\text {cde }}$ \\
\hline & $\begin{array}{c}\text { Tomato + } \\
\text { sesame oil cake }\end{array}$ & $39.9^{a b}$ & $17.2^{\mathrm{a}}$ & $12.6^{\text {abcde }}$ & $20.7^{\mathrm{a}}$ & $47.6^{\mathrm{a}}$ \\
\hline & Onion + fish & $14.2^{b c d}$ & $2.6^{\mathrm{ab}}$ & $24.6^{\mathrm{abc}}$ & $12.1^{\mathrm{abc}}$ & $35.5^{\mathrm{abc}}$ \\
\hline & $\begin{array}{l}\text { Onion + sesame } \\
\text { oil cake }\end{array}$ & $28.1^{\mathrm{abcd}}$ & $17.2^{\mathrm{a}}$ & 15.9 abcde & $9.2^{\mathrm{abc}}$ & $20.7^{\text {cde }}$ \\
\hline
\end{tabular}

DAT, days after treatment. * Means within a column followed by the same letters are not significantly different at the $5 \%$ level according to Duncan's Multiple Range Test. 
At 14 days after the treatments, plant height increased significantly after being treated with tomato + fish and tomato + sesame oil cake fermentation extract combinations; tomato + sesame oil cake boiled water extract combination; and onion + fish water extract combination, compared to the control. Cucumber leaf number increased significantly after being treated with a combination of tomato + sesame oil cake water extracts, compared to the control. Cucumber shoot fresh weight increased significantly after being treated with a combination of tomato + fish fermentation extracts, boiled water extracts made using tomato + fish and tomato + sesame oil cake, and water extracts made using tomato + sesame oil cake and onion + fish, compared to the control. On the other hand, although these combinations increased plant height, leaf number, and shoot fresh weight compared to the control, they did not produce increases that were significantly greater than those observed in the cucumber plants that had received applications of single material extracts. This result means that when separate extracts were combined, they did not produce any sort of extra growth-promoting effect. Therefore, in order to find ways of improving extracts' growth promotion effects, our future studies will examine how natural emulsifiers, when combined with extracts, might produce higher rates of growth promotion.

To confirm the growth promotion effects, plant height and leaf number were measured at 20 and 40 days after the treatments and fruit length and fruit weight at harvest after various extract treatments (Table 3). At 20 days after the treatments, plant height increased significantly when treated with the fermentation bone + fish, tomato, onion, and red pepper leaf extracts; boiled water onion extract; and water bone + fish, onion, and sesame oil cake extracts compared to the control. On the other hand, the aforementioned extracts produced similar increases in plant height compared to oil cake. Forty days after the treatments, plant height increased significantly after treatments of all extracts compared to the control. In addition, plant height increased significantly after the treatments of most extracts compared to the control. On the other hand, regardless of which extract had been applied, the leaf number at 20 or 40 days after treatments was similar to the control or oil cake. Fruit length at harvest was 17-42\%, 15-43\%, and 19-32\% higher than the control when the plants were treated with the fermentation, boiled water, and water extracts, respectively. Furthermore, fruit length increased significantly after the treatments of fermentation bone + fish, tomato, and red pepper leaf extracts; boiled water bone + fish and onion extracts; and water bone + fish, onion, and sesame oil cake extracts compared to the oil cake. The fruit weight was $17-81 \%, 10-61 \%$, and $10-45 \%$ higher than the control or oil cake when the plants were treated with the fermentation, boiled water, and water extracts, respectively. Although the extraction methods for liquid fertilizers can result in different contents or physiological activities of active substances, extraction methods of various materials used in this study showed similar cucumber growth and yield promotion effects. Fermentation extraction is one of the most common methods used by organic farmers. On the other hand, fermentation extracts have a longer manufacturing period than those that are produced using other extraction methods. Thus, boiled water and water extracts are more suitable for organic cultivation because they are both good growth promotors and, unlike fermentation extracts, can be produced in a short time. 
Table 3. Effect of various extracts on plant height, leaf number, fruit length, and fruit weight of cucumber using drenching application at $1 \%$ concentrations.

\begin{tabular}{|c|c|c|c|c|c|c|c|}
\hline \multirow{2}{*}{$\begin{array}{l}\text { Extraction } \\
\text { Method }\end{array}$} & \multirow[b]{2}{*}{ Extract } & \multicolumn{2}{|c|}{20 DAT (Once Appl.) } & \multicolumn{2}{|c|}{40 DAT (Twice Appl.) } & \multicolumn{2}{|c|}{ Harvest (Four Appl.) } \\
\hline & & $\begin{array}{c}\text { Plant } \\
\text { Height }\end{array}$ & $\begin{array}{c}\text { Leaf } \\
\text { Number }\end{array}$ & $\begin{array}{c}\text { Plant } \\
\text { Height }\end{array}$ & $\begin{array}{c}\text { Leaf } \\
\text { Number }\end{array}$ & Fruit Length & Fruit Weight \\
\hline & & \multicolumn{6}{|c|}{ — of Control- } \\
\hline & Control & $0.0^{\mathrm{d} *}$ & $0.0^{\mathrm{a}}$ & $0.0 \mathrm{~g}$ & $0.0^{\mathrm{ab}}$ & $0.0^{\mathrm{h}}$ & $0.0 \mathrm{~g}$ \\
\hline & Oil cake & $13.2^{a b c}$ & $10.8^{\mathrm{a}}$ & $13.8^{\mathrm{e}}$ & $-0.4^{\mathrm{ab}}$ & $18.2^{\mathrm{fg}}$ & $1.0 \mathrm{~g}$ \\
\hline \multirow{5}{*}{ Fermentation } & Bone + fish meal & $8.9^{c}$ & $3.8^{\mathrm{a}}$ & $24.1^{\mathrm{b}}$ & $16.8^{a b}$ & $30.3^{\mathrm{cd}}$ & $16.9^{\mathrm{f}}$ \\
\hline & Tomato & $20.5^{a}$ & $7.3^{\mathrm{a}}$ & $18.1^{\mathrm{d}}$ & $11.4^{\mathrm{ab}}$ & $42.3^{b}$ & $80.5^{b}$ \\
\hline & Onion & $14.2^{b c}$ & $7.3^{\mathrm{a}}$ & $18.1^{\mathrm{d}}$ & $14.1^{\mathrm{ab}}$ & $18.9 \mathrm{fg}$ & $-4.1^{\mathrm{g}}$ \\
\hline & Red pepper leaf & $17.1^{\mathrm{abc}}$ & $7.3^{\mathrm{a}}$ & $18.5^{\mathrm{d}}$ & $14.1^{\mathrm{ab}}$ & $41.8^{\mathrm{b}}$ & $49.1^{\mathrm{d}}$ \\
\hline & Sesame oil cake & $3.2^{\mathrm{d}}$ & $0.4^{\mathrm{a}}$ & $17.8^{\mathrm{d}}$ & $5.1^{\mathrm{ab}}$ & $17.3^{\mathrm{g}}$ & $-2.5^{\mathrm{g}}$ \\
\hline \multirow{5}{*}{ Boiled water } & Bone + fish meal & $-3.2^{\mathrm{d}}$ & $6.2^{a}$ & $23.7^{\mathrm{b}}$ & $16.8^{a b}$ & $42.9^{b}$ & $60.5^{c}$ \\
\hline & Tomato & $3.8^{\mathrm{d}}$ & $0.4^{\mathrm{a}}$ & $22.6^{\mathrm{bc}}$ & $19.6^{\mathrm{a}}$ & $23.6^{\text {ef }}$ & $34.0^{\mathrm{e}}$ \\
\hline & Onion & $14.8^{\mathrm{abc}}$ & $6.2^{\mathrm{a}}$ & $34.1^{\mathrm{a}}$ & $22.3^{a}$ & $32.0^{\mathrm{c}}$ & $34.0^{\mathrm{e}}$ \\
\hline & Red pepper leaf & $3.5^{\mathrm{d}}$ & $0.4^{\mathrm{a}}$ & $17.0^{\mathrm{cd}}$ & $11.4^{\mathrm{ab}}$ & $19.4^{\mathrm{fg}}$ & $10.4^{\mathrm{f}}$ \\
\hline & Sesame oil cake & $0.6^{\mathrm{d}}$ & $0.4^{\mathrm{a}}$ & $15.9^{\mathrm{d}}$ & $19.6^{a}$ & $14.9^{g}$ & $1.2 \mathrm{~g}$ \\
\hline \multirow{5}{*}{ Water } & Bone + fish meal & $11.6^{b c}$ & $1.5^{\mathrm{a}}$ & $6.7^{f}$ & $-5.8^{b}$ & $26.3^{\mathrm{cd}}$ & $29.9^{\mathrm{e}}$ \\
\hline & Tomato & $3.8^{\mathrm{d}}$ & $0.4^{\mathrm{a}}$ & $22.6^{\mathrm{bc}}$ & $19.6^{a}$ & $23.6^{\text {ef }}$ & $34.0^{\mathrm{e}}$ \\
\hline & Onion & $14.8^{a b c}$ & $6.2^{a}$ & $34.1^{\mathrm{a}}$ & $22.3^{a}$ & $32.0^{c}$ & $34.0^{\mathrm{e}}$ \\
\hline & Red pepper leaf & $3.5^{\mathrm{d}}$ & $0.4^{\mathrm{a}}$ & $17.0^{\mathrm{cd}}$ & $11.4^{\mathrm{ab}}$ & $19.4^{\mathrm{fg}}$ & $10.4^{\mathrm{f}}$ \\
\hline & Sesame oil cake & $14.2^{\mathrm{abc}}$ & $3.8^{a}$ & 8.9 ef & $5.1^{\mathrm{ab}}$ & 27.7 be & $45.3^{\mathrm{d}}$ \\
\hline
\end{tabular}

DAT, days after treatment; appl., application. * Means within a column followed by the same letters are not significantly different at the $5 \%$ level according to Duncan's Multiple Range Test.

\subsection{Total Chlorophyll Reading (SPAD) and Photosynthetic Efficacy}

At 7 days after the treatments, compared to the control, chlorophyll content (SPAD values) increased significantly in plants treated with the fermentation tomato and sesame oil cake extracts and the water bone + fish, onion, and red pepper leaf extracts (Table 4). On the other hand, chlorophyll content did not vary significantly in the plants treated with most extracts compared to the control. In addition, the photosynthetic efficiency of the cucumber plants treated with various extracts did not vary significantly. At 14 days after the treatments, chlorophyll content and photosynthetic efficiency were also similar in both the treated and control plants.

In studies where plants were treated with either a single material extract or a mixture of extracts, chlorophyll content was increased significantly 7 days after treatment only in the plants treated with the tomato and onion + fish extracts compared to the control (Table 5). Photosynthetic efficiency did not vary significantly regardless of which extract had been applied. At 14 days after the treatments, chlorophyll content and photosynthetic efficiency were also similar in both the treated and control plants. In other studies, there were no significant differences in photosynthetic efficiency, chlorophyll, or carotenoid contents in lettuce and Peucedanum japonicum Thunb. plants treated with Chinese chive, soybean leaf, soybean stem, onion, and tomato water extracts and the untreated control [30,31]. Other studies, however, suggested that an increase in chlorophyll content could enhance the photosynthesis rates, which ultimately influence maize growth [34]. Phaseolus vulgaris plants sprayed with Moringa oleifera leaf extract increased significantly in chlorophyll content compared to the environmental stresses [35]. Furthermore, one of the characteristic 
responses to seaweed extract treatments is an increase in chlorophyll content in the treated plants, which has been observed in grapevine and strawberry $[12,14,36]$. What these studies show is that photosynthetic efficiency and chlorophyll contents increases only when certain plant extracts are applied to certain plants. In other cases, extract treatments have little to no effect on photosynthetic efficiency and chlorophyll contents. Other mechanisms which may cause these sorts of changes are higher mineral nutrient contents in the plant extracts themselves and the nutrient uptake in the treated leaves. Further studies are needed in order to better understand how these mechanisms may affect growth promotion.

Table 4. Effect of various plant extracts on SPAD values (chlorophyll content) and quantum yield (photosynthesis efficacy) in cucumber plants at 7 and 14 days after treatment (DAT) using drenching application at $1 \%$ concentrations.

\begin{tabular}{|c|c|c|c|c|c|c|}
\hline \multirow{2}{*}{$\begin{array}{c}\text { Extraction } \\
\text { Method }\end{array}$} & \multirow{2}{*}{\multicolumn{2}{|c|}{ Extract/Application Frequency }} & \multicolumn{2}{|c|}{7 DAT } & \multicolumn{2}{|c|}{14 DAT } \\
\hline & & & SPAD Values & Quantum Yield & SPAD Values & Quantum Yield \\
\hline & \multicolumn{2}{|c|}{ Control } & $26.57^{\mathrm{efg} *}$ & $0.81^{\text {abcd }}$ & $28.40^{a b c}$ & $0.80^{b}$ \\
\hline \multirow{12}{*}{ Fermentation } & \multirow[b]{2}{*}{ Fish } & Once & $27.13^{\text {cdefg }}$ & 0.80 cdef & $29.00^{a b c}$ & $0.80^{b}$ \\
\hline & & Twice & $28.90^{\text {abcdef }}$ & $0.80^{\text {cdef }}$ & $29.17^{a b c}$ & $0.80^{b}$ \\
\hline & \multirow[b]{2}{*}{ Bone + fish meal } & Once & $27.13^{\text {cdefg }}$ & $0.81^{\text {abcd }}$ & $29.47^{\mathrm{abc}}$ & $0.80^{\mathrm{b}}$ \\
\hline & & Twice & $27.57^{\text {bcdefg }}$ & 0.81 abcde & $30.33^{a b c}$ & $0.80^{b}$ \\
\hline & \multirow{2}{*}{ Tomato } & Once & $29.13^{\text {abcdef }}$ & $0.78^{\mathrm{f}}$ & $30.93^{a b}$ & $0.80^{b}$ \\
\hline & & Twice & $30.00^{a b c}$ & $0.80^{\text {bcdef }}$ & $30.76^{a b c}$ & $0.81^{b}$ \\
\hline & \multirow{2}{*}{ Onion } & Once & $27.03^{\text {defg }}$ & $0.80^{\text {cdef }}$ & $28.90^{a b c}$ & $0.80^{\mathrm{b}}$ \\
\hline & & Twice & 28.22 bcdefg & $0.82^{a b c}$ & $28.80^{a b c}$ & $0.81^{a b}$ \\
\hline & \multirow{2}{*}{ Red pepper leaf } & Once & $29.63^{\mathrm{abcd}}$ & $0.80^{\text {cdef }}$ & $30.97^{a b}$ & $0.80^{\mathrm{b}}$ \\
\hline & & Twice & $27.77^{\text {bcdefg }}$ & $0.81^{\mathrm{abcd}}$ & $29.80^{a b c}$ & $0.81^{\mathrm{b}}$ \\
\hline & \multirow{2}{*}{ Sesame oil cake } & Once & $31.62^{\mathrm{a}}$ & 0.79 ef & $29.00 \mathrm{abc}$ & $0.83^{\mathrm{a}}$ \\
\hline & & Twice & $28.91^{\text {abcdef }}$ & $0.80^{\mathrm{cdef}}$ & $31.03^{a}$ & $0.80^{b}$ \\
\hline \multirow{12}{*}{ Boiled water } & \multirow{2}{*}{ Fish } & Once & $27.67^{\text {bcdefg }}$ & $0.83^{\mathrm{a}}$ & $29.00^{a b c}$ & $0.79^{b}$ \\
\hline & & Twice & $29.37^{\text {abcde }}$ & 0.81 abcde & $30.97^{a b}$ & $0.80^{b}$ \\
\hline & \multirow{2}{*}{ Bone + fish meal } & Once & 28.20 bcdefg & 0.79 def & $29.53^{a b c}$ & $0.80^{b}$ \\
\hline & & Twice & $26.43^{\mathrm{fg}}$ & $0.80^{\text {bcdef }}$ & $28.10^{a b c}$ & $0.80^{b}$ \\
\hline & \multirow{2}{*}{ Tomato } & Once & $26.30 \mathrm{fg}$ & 0.80 bcdef & $28.97^{a b c}$ & $0.80^{b}$ \\
\hline & & Twice & $27.70^{\text {bcdefg }}$ & $0.80^{\text {cdef }}$ & $27.53^{c}$ & $0.80^{b}$ \\
\hline & \multirow{2}{*}{ Onion } & Once & 29.00 abcdef & 0.81 abcde & $29.07^{a b c}$ & $0.80^{b}$ \\
\hline & & Twice & $27.30^{\text {bcdefg }}$ & $0.82^{\mathrm{ab}}$ & $28.73^{a b c}$ & $0.81^{b}$ \\
\hline & \multirow{2}{*}{ Red pepper leaf } & Once & $27.87^{\text {bcdefg }}$ & 0.79 def & $28.80^{a b c}$ & $0.80^{b}$ \\
\hline & & Twice & $28.77^{\text {bcdef }}$ & $0.81^{\mathrm{abcd}}$ & $29.17^{a b c}$ & $0.80^{b}$ \\
\hline & \multirow{2}{*}{ Sesame oil cake } & Once & $28.13^{\text {bcdefg }}$ & 0.79 ef & $30.73^{a b c}$ & $0.79^{b}$ \\
\hline & & Twice & $29.10^{\text {abcdef }}$ & $0.80^{\text {bcdef }}$ & $30.03^{a b c}$ & $0.81^{\mathrm{ab}}$ \\
\hline \multirow{12}{*}{ Water } & \multirow{2}{*}{ Fish } & Once & 29.80 abcd & 0.79 def & $29.47^{a b c}$ & $0.80^{b}$ \\
\hline & & Twice & $29.10^{\text {abcdef }}$ & 0.81 abcde & $30.97^{a b}$ & $0.80^{b}$ \\
\hline & \multirow{2}{*}{ Bone + fish meal } & Once & $30.07^{a b}$ & 0.81 abcde & $29.70^{a b c}$ & $0.80^{b}$ \\
\hline & & Twice & $28.43^{\text {bcdefg }}$ & $0.80^{\text {bcdef }}$ & $28.10^{a b c}$ & $0.80^{b}$ \\
\hline & \multirow{2}{*}{ Tomato } & Once & $27.47^{\text {bcdefg }}$ & $0.80^{\text {cdef }}$ & $27.70^{b c}$ & $0.80^{b}$ \\
\hline & & Twice & $29.97^{a b c}$ & $0.80^{\text {bcdef }}$ & $27.53^{c}$ & $0.80^{b}$ \\
\hline & \multirow{2}{*}{ Onion } & Once & $25.90^{g}$ & $0.80^{\text {bcdef }}$ & $27.97^{a b c}$ & $0.79^{b}$ \\
\hline & & Twice & $29.60^{a b c d}$ & $0.82^{a b c}$ & $28.73^{a b c}$ & $0.79^{b}$ \\
\hline & \multirow{2}{*}{ Red pepper leaf } & Once & $27.93^{\text {bcdefg }}$ & 0.80 cdef & $28.20^{a b c}$ & $0.80^{b}$ \\
\hline & & Twice & $30.00^{\mathrm{abc}}$ & $0.81^{\text {abcd }}$ & $29.17^{a b c}$ & $0.79^{b}$ \\
\hline & \multirow{2}{*}{ Sesame oil cake } & Once & $28.13^{\text {bcdefg }}$ & $0.80^{\text {cdef }}$ & $28.30^{a b c}$ & $0.80^{b}$ \\
\hline & & Twice & $29.10^{\text {abcdef }}$ & $0.80^{\text {bcdef }}$ & $30.03^{a b c}$ & $0.80^{b}$ \\
\hline
\end{tabular}

* Means within a column followed by the same letters are not significantly different at the 5\% level according to Duncan's Multiple Range Test. 
Table 5. Effect of combined extracts on SPAD values (chlorophyll content) and quantum yield (photosynthesis efficacy) in cucumber plants at 7 and 14 days after treatment (DAT) using drenching application at $1 \%$ concentrations.

\begin{tabular}{|c|c|c|c|c|c|}
\hline \multirow{2}{*}{$\begin{array}{l}\text { Extraction } \\
\text { Method }\end{array}$} & \multirow{2}{*}{ Extract } & \multicolumn{2}{|c|}{7 DAT } & \multicolumn{2}{|c|}{14 DAT } \\
\hline & & SPAD Values & Quantum Yield & SPAD Values & Quantum Yield \\
\hline & & \multicolumn{4}{|c|}{ \% of Control } \\
\hline & Control & $33.4^{\text {bcde } *}$ & $0.81^{\mathrm{b}}$ & $29.4^{a b c}$ & $0.81^{\mathrm{b}}$ \\
\hline \multirow{8}{*}{ Fermentation } & Tomato & $34.4^{\mathrm{abc}}$ & $0.82^{a b}$ & $29.3^{a b c}$ & $0.82^{a b}$ \\
\hline & Onion & $35.1^{\mathrm{ab}}$ & $0.82^{a b}$ & $31.2^{\mathrm{ab}}$ & $0.82^{a b}$ \\
\hline & Fish & $34.4^{\mathrm{ab}}$ & $0.82^{a b}$ & $30.9^{\mathrm{abc}}$ & $0.83^{a b}$ \\
\hline & Sesame oil cake & $35.1^{\mathrm{ab}}$ & $0.82^{a b}$ & $30.7^{a b c}$ & $0.81 \mathrm{ab}$ \\
\hline & Tomato + fish & $33.8^{\text {abcde }}$ & $0.82^{\mathrm{a}}$ & $33.6^{\mathrm{a}}$ & $0.81^{\mathrm{b}}$ \\
\hline & $\begin{array}{c}\text { Tomato + sesame oil } \\
\text { cake }\end{array}$ & 32.8 bcde & $0.82^{\mathrm{a}}$ & $26.6^{c}$ & $0.82^{\mathrm{ab}}$ \\
\hline & Onion + fish & 31.7 de & $0.82^{a b}$ & $28.2^{b c}$ & $0.82^{a b}$ \\
\hline & Onion + sesame oil cake & $33.1^{\text {bcde }}$ & $0.82^{\mathrm{a}}$ & $27.4^{\text {bc }}$ & $0.81^{\mathrm{b}}$ \\
\hline \multirow{8}{*}{ Boiled water } & Tomato & $33.1^{\text {bcde }}$ & $0.82^{a b}$ & $28.6^{\mathrm{bc}}$ & $0.84^{\mathrm{ab}}$ \\
\hline & Onion & 33.3 bcde & $0.82^{\mathrm{a}}$ & $27.8^{\mathrm{bc}}$ & $0.82^{a b}$ \\
\hline & Fish & $35.3^{a b}$ & $0.82^{a b}$ & $27.9 \mathrm{bc}$ & $0.83^{\mathrm{ab}}$ \\
\hline & Sesame oil cake & $31.3^{\mathrm{e}}$ & $0.82^{a b}$ & $28.8^{b c}$ & $0.82^{a b}$ \\
\hline & Tomato + fish & 34.0 abcde & $0.81^{\mathrm{ab}}$ & $29.0^{a b c}$ & $0.81^{\mathrm{ab}}$ \\
\hline & $\begin{array}{c}\text { Tomato + sesame oil } \\
\text { cake }\end{array}$ & $35.3^{\mathrm{ab}}$ & $0.82^{a b}$ & $31.4^{\mathrm{ab}}$ & $0.81^{b}$ \\
\hline & Onion + fish & $34.3^{\text {abcd }}$ & $0.82^{a b}$ & $28.4^{b c}$ & $0.82 \mathrm{ab}$ \\
\hline & Onion + sesame oil cake & $33.6^{\text {abcde }}$ & $0.82^{\mathrm{a}}$ & $30.1^{\mathrm{abc}}$ & $0.82^{a b}$ \\
\hline \multirow{8}{*}{ Water } & Tomato & $36.3^{a}$ & $0.82^{\mathrm{a}}$ & $28.7^{b c}$ & $0.81^{\mathrm{ab}}$ \\
\hline & Onion & $34.8^{\mathrm{ab}}$ & $0.82^{a b}$ & $28.5^{b c}$ & $0.82^{a b}$ \\
\hline & Fish & $31.7^{\text {cde }}$ & $0.82^{a b}$ & $29.4^{\mathrm{abc}}$ & $0.82^{a b}$ \\
\hline & Sesame oil cake & $35.0^{\mathrm{ab}}$ & $0.82^{a b}$ & $28.7^{b c}$ & $0.82^{a b}$ \\
\hline & Tomato + fish & 33.4 bcde & $0.82^{a b}$ & $28.2^{b c}$ & $0.82^{a b}$ \\
\hline & $\begin{array}{c}\text { Tomato + sesame oil } \\
\text { cake }\end{array}$ & $35.6^{\mathrm{ab}}$ & $0.82^{a}$ & $31.3^{\mathrm{ab}}$ & $0.82^{a b}$ \\
\hline & Onion + fish & $36.2^{\mathrm{a}}$ & $0.82^{a b}$ & $30.2^{a b c}$ & $0.86^{\mathrm{a}}$ \\
\hline & Onion + sesame oil cake & $33.3^{\text {bcde }}$ & $0.82^{\mathrm{a}}$ & $30.6^{a b c}$ & $0.81^{b}$ \\
\hline
\end{tabular}

* Means within a column followed by the same letters are not significantly different at the $5 \%$ level according to Duncan's Multiple Range Test.

\subsection{Levels of Mineral Nutrition in Extracts and Treated Leaves}

Among the fermentation extracts, the fish extracts contained the highest levels of $\mathrm{K}, \mathrm{Ca}$, and $\mathrm{Mg}$ and some of the highest levels of $\mathrm{N}$ and $\mathrm{P}$ (Table 6). In addition, the fermentation bone + fish extracts contained the second-highest levels of macro elements: $\mathrm{N}, \mathrm{K}, \mathrm{Ca}, \mathrm{Mg}$, and $\mathrm{Na}$. Among the boiled water extracts, the fish extracts contained the highest levels of $\mathrm{N}$ and $\mathrm{P}$; the tomato extracts contained the highest levels of $\mathrm{K}$ and $\mathrm{Na}$, and the red pepper extracts contained the highest levels of $\mathrm{Ca}$ and $\mathrm{Mg}$. Among the water extracts, the fish extracts contained the highest levels of $\mathrm{N}$ and $\mathrm{Na}$, and bones + fish extracts contained the highest levels of $\mathrm{P}, \mathrm{Ca}$, and $\mathrm{Mg}$. Generally, among all the different extracts, those extracted using the boiled water method had greater levels of $\mathrm{N}$ than those produced using fermentation and water methods. On the other hand, the Ca content was greater in fermentation extracts than boiled water and water extracts. The $\mathrm{P}$ and $\mathrm{Na}$ contents in the water extracts were higher than in the fermentation and boiled water extracts. On the other hand, the $\mathrm{K}$ and $\mathrm{Mg}$ contents were similar regardless of which extraction method was used. 
Table 6. Nutrition levels of various extracts.

\begin{tabular}{|c|c|c|c|c|c|c|c|c|c|c|c|c|}
\hline \multirow{2}{*}{$\begin{array}{l}\text { Extraction } \\
\text { Method }\end{array}$} & \multirow[b]{2}{*}{ Extract } & \multicolumn{6}{|c|}{ Macro Elements } & \multicolumn{5}{|c|}{ Micro Elements (mg/kg) } \\
\hline & & N (\%) & $\begin{array}{c}\mathrm{P} \\
(\mathrm{mg} / \mathrm{kg})\end{array}$ & $\begin{array}{c}\mathrm{K} \\
(\mathrm{mg} / \mathrm{kg})\end{array}$ & $\begin{array}{c}\mathrm{Ca} \\
(\mathrm{mg} / \mathrm{kg})\end{array}$ & $\underset{\text { (mg/kg) }}{\mathrm{Mg}}$ & $\underset{(\mathrm{mg} / \mathrm{kg})}{\mathrm{Na}}$ & $\mathrm{Cu}$ & Zn & $\mathrm{Fe}$ & Mn & B \\
\hline \multirow{6}{*}{ Fermentation } & Fish & $1.84^{b *}$ & $130.6^{b}$ & $31426.6^{a}$ & $11273.6^{\mathrm{a}}$ & $1037.6^{a}$ & $867.8^{\text {ef }}$ & $0.24^{c}$ & $2.56^{\mathrm{ef}}$ & $10.76^{\mathrm{g}}$ & 0.00 & $2.74 \mathrm{~g}$ \\
\hline & $\begin{array}{l}\text { Bone }+ \text { fish } \\
\text { meal }\end{array}$ & $0.56^{\mathrm{hi}}$ & $40.6^{\mathrm{g}}$ & $3259.4^{\mathrm{k}}$ & $4357.4^{\mathrm{b}}$ & $1003.4^{b}$ & $2443.8^{\mathrm{d}}$ & $0.00^{\mathrm{c}}$ & $0.14^{\mathrm{k}}$ & $6.12^{\mathrm{i}}$ & 0.02 & $2.28^{\mathrm{ijk}}$ \\
\hline & Tomato & $0.10^{1}$ & $28.0^{\mathrm{ij}}$ & $1662.6^{\mathrm{n}}$ & $140.0^{1}$ & $32.0^{\circ}$ & $196.8^{\mathrm{h}}$ & $0.18^{c}$ & $1.84 \mathrm{fgh}$ & $21.40^{\mathrm{b}}$ & ND & $2.20 \mathrm{ijk}$ \\
\hline & Onion & $0.06^{\mathrm{m}}$ & $40.6^{\mathrm{g}}$ & $1184.2^{\circ}$ & $6.6^{\mathrm{n}}$ & $30.8^{\circ}$ & $185.4^{\mathrm{h}}$ & $0.00^{c}$ & $0.44^{\mathrm{jk}}$ & $8.80^{\mathrm{h}}$ & 0.00 & $2.28^{\mathrm{ijk}}$ \\
\hline & Red pepper leaf & $0.02^{\mathrm{n}}$ & $16.0^{1}$ & $2810.2^{1}$ & $0.2^{n}$ & $131.0^{\mathrm{m}}$ & $379.2^{\mathrm{g}}$ & $0.00^{c}$ & $0.14^{\mathrm{k}}$ & $5.96^{\mathrm{i}}$ & 0.00 & $2.50^{\mathrm{h}}$ \\
\hline & Sesame oil cake & $0.46^{\mathrm{j}}$ & $62.0^{\mathrm{f}}$ & $2876.6^{1}$ & $305.2 \mathrm{gh}$ & $804.6^{\mathrm{f}}$ & $5500.0^{\mathrm{b}}$ & $0.02^{\mathrm{c}}$ & $1.10^{\mathrm{hij}}$ & $19.98^{c}$ & 0.00 & $2.72^{g}$ \\
\hline \multirow{7}{*}{ Boiled water } & Fish & $2.64^{\mathrm{a}}$ & $104.6^{c}$ & $4601.0^{\mathrm{j}}$ & $53.2^{\mathrm{mn}}$ & $2.2^{p}$ & $864.0^{\text {ef }}$ & $0.04^{c}$ & $0.68^{\mathrm{ijk}}$ & $2.36^{\mathrm{k}}$ & ND & $2.36^{\mathrm{hi}}$ \\
\hline & Bone meal & $0.54^{\mathrm{i}}$ & $60.0^{f}$ & $805.4^{\mathrm{p}}$ & $205.8^{\mathrm{jk}}$ & $168.8^{1}$ & $864.0^{\text {ef }}$ & $0.30^{\mathrm{c}}$ & $1.14^{\text {hij }}$ & $4.26^{j}$ & ND & $2.12^{\mathrm{k}}$ \\
\hline & Fish meal & $0.34^{\mathrm{k}}$ & $37.2^{\mathrm{gh}}$ & $2086.0^{\mathrm{m}}$ & $336.8^{\mathrm{g}}$ & $439.6^{j}$ & $696.0^{f}$ & $1.02^{\mathrm{c}}$ & $7.08^{c}$ & $18.76^{\mathrm{d}}$ & 0.00 & $2.30^{\mathrm{ij}}$ \\
\hline & Tomato & $0.74 \mathrm{~g}$ & $23.2^{j \mathrm{k}}$ & $15361.0^{\mathrm{c}}$ & $281.0^{\mathrm{hi}}$ & $567.0 \mathrm{~g}$ & $922.8^{\text {e }}$ & $0.96^{c}$ & $12.36^{\mathrm{b}}$ & $32.90^{a}$ & 0.00 & $3.50^{\mathrm{d}}$ \\
\hline & Onion & $0.80^{f}$ & $8.0^{\mathrm{m}}$ & $7325.8^{\mathrm{g}}$ & $443.6^{\text {ef }}$ & $480.2^{i}$ & $926.6^{\mathrm{e}}$ & $0.28^{c}$ & $5.50^{\mathrm{d}}$ & $8.68^{h}$ & 0.00 & $3.28^{\text {ef }}$ \\
\hline & Red pepper leaf & $1.16^{\mathrm{d}}$ & $32.0^{\text {hi }}$ & $9421.6^{\mathrm{d}}$ & $1747.2^{\mathrm{c}}$ & $1035.2^{\mathrm{a}}$ & $411.6^{\mathrm{g}}$ & $3.72^{b c}$ & $16.16^{\mathrm{a}}$ & $18.14^{\mathrm{d}}$ & 0.14 & $4.98^{b}$ \\
\hline & Sesame oil cake & $0.58^{\mathrm{h}}$ & $73.2^{\mathrm{e}}$ & $5540.6^{\mathrm{h}}$ & $399.2^{f}$ & $925.8^{\mathrm{d}}$ & $151.8^{\mathrm{h}}$ & $0.64^{c}$ & $2.77^{\mathrm{d}}$ & $8.36^{\mathrm{h}}$ & 0.00 & $5.20^{\mathrm{a}}$ \\
\hline \multirow{7}{*}{ Water } & Fish & $1.72^{\mathrm{c}}$ & $74.6^{\mathrm{e}}$ & $5058.8^{\mathrm{i}}$ & $162.8^{\mathrm{kl}}$ & $2.4^{\mathrm{P}}$ & $11275.8^{a}$ & $0.22^{c}$ & 1.38 ghi & $11.16^{\mathrm{g}}$ & 0.02 & $2.30^{\mathrm{ij}}$ \\
\hline & Bone meal & $0.48^{\mathrm{j}}$ & $26.6^{\mathrm{ijk}}$ & $636.0^{\mathrm{p}}$ & $161.6^{\mathrm{kl}}$ & $88.4^{n}$ & $3183.6^{c}$ & $0.00^{c}$ & $0.64^{\mathrm{ijk}}$ & $17.42^{\mathrm{e}}$ & ND & $2.18^{\mathrm{jk}}$ \\
\hline & Fish meal & $0.46^{\mathrm{j}}$ & $168.6^{a}$ & $5558.4^{\mathrm{h}}$ & $860.0^{d}$ & $1010.4^{b}$ & $2372.6^{\mathrm{d}}$ & $1.06^{\mathrm{c}}$ & $11.80^{\mathrm{b}}$ & $16.42^{f}$ & 0.00 & $3.20^{f}$ \\
\hline & Tomato & $0.88^{\mathrm{e}}$ & $81.2^{\mathrm{d}}$ & $17436.6^{\mathrm{b}}$ & $64.6^{\mathrm{m}}$ & $544.0^{\mathrm{h}}$ & $824.0^{\text {ef }}$ & $8.64^{\mathrm{a}}$ & $11.76^{\mathrm{b}}$ & $20.84^{\mathrm{b}}$ & 0.00 & $3.52^{\mathrm{d}}$ \\
\hline & Onion & $0.86^{\mathrm{e}}$ & $20.6^{\mathrm{kl}}$ & $8056.8^{f}$ & $209.8^{j k}$ & $347.0^{\mathrm{k}}$ & 831.2 ef & $2.02^{b c}$ & $5.42^{\mathrm{d}}$ & $16.42^{f}$ & 0.00 & $3.40^{\mathrm{de}}$ \\
\hline & Red pepper leaf & $0.72 \mathrm{~g}$ & $81.2^{\mathrm{d}}$ & $8251.2^{\text {e }}$ & $235.8^{\mathrm{ij}}$ & $979.6^{c}$ & $265.4^{\mathrm{gh}}$ & $5.10^{\mathrm{b}}$ & $3.02 \mathrm{e}$ & $6.54^{\mathrm{i}}$ & 0.06 & $3.50^{\mathrm{d}}$ \\
\hline & Sesame oil cake & $0.88^{\mathrm{e}}$ & $100.6^{c}$ & $5068.2^{i}$ & $475.6^{\mathrm{e}}$ & $873.4^{\mathrm{e}}$ & $114.4^{\mathrm{h}}$ & $0.56^{c}$ & $2.18^{\text {efg }}$ & $6.64^{\mathrm{i}}$ & 0.00 & $4.32^{c}$ \\
\hline
\end{tabular}

* Means within a column followed by the same letters are not significantly different at the $5 \%$ level according to Duncan's Multiple Range Test.

Regarding the micro elements and among all the fermentation extracts, the fish extracts contained the highest levels of $\mathrm{Ca}$ and $\mathrm{Zn}$; the tomato extracts contained the highest levels of Fe, and sesame oil cake extracts contained the highest levels of B. Among the boiled water extracts, the tomato extracts contained the highest levels of Fe; the red pepper leaf extracts contained the highest levels of $\mathrm{Cu}$ and $\mathrm{Zn}$, and the sesame oil cake extract contained the highest levels of B. Among the water extracts, the tomato extracts contained the highest levels of $\mathrm{Cu}, \mathrm{Zn}$, and $\mathrm{Fe}$, and the sesame oil cake extracts contained the highest levels of B. Overall, the extracts produced using water extraction methods had greater $\mathrm{Cu}$ contents than the fermentation and boiled water extracts. The $\mathrm{Zn}$ contents in the boiled water and water extracts were higher than the fermentation extracts. On the other hand, the Fe contents were similar regardless of which extraction method had been used. In this study, however, the extracts with the highest levels of mineral nutrients were not the most effective growth promotors of cucumber plants. Previous studies also showed that higher levels of macro and micro elements are not solely responsible for growth promotion $[6,30]$. This suggests that cucumber growth and yield promotion might not be caused by high levels of one specific macro or micro element but rather by a specific combination of various elements. In addition, the increases in growth traits may be attributed to the high levels of growth-promoting hormones auxins, gibberellins, and cytokinins.

The nutrient levels in the cucumber leaves were also measured after the extract treatments (Table 7). Plants absorb nutrients, either through the roots or from the leaf surface. The extracts alter the physical, biochemical, and biological properties of the soil and may affect the architecture of the plant roots facilitating the efficient uptake of nutrients [37]. The total $\mathrm{N}$ level in the cucumber leaves was similar when treated with the fermentation red pepper leaf and sesame oil cake extracts, boiled water red pepper extracts, water onion, and sesame oil cake extracts compared to the control. On the other hand, the total $\mathrm{N}$ in the cucumber leaves decreased significantly when treated with most other extracts compared to the control. The P contents increased significantly when treated with the boiled water bone + fish, tomato, and red pepper leaf, and water sesame oil cake extracts compared to the control. In addition, the boiled water onion and sesame oil cake, water bone + fish, tomato, onion, and red pepper leaf extracts did not increase the P levels significantly. In contrast, the $\mathrm{P}$ contents decreased significantly when treated with all the fermentation extracts tested compared to the control. The $\mathrm{K}$ contents were similar when treated with the fermentation sesame oil cake, water bone + fish and sesame oil cake extracts compared to 
the control. The $\mathrm{K}$ contents decreased significantly when treated with most other extracts compared to the control. The Ca contents increased significantly when treated with just the boiled water bone + fish and water bone + fish extracts compared to the control. The Ca contents decreased significantly when treated with most extracts compared to the control. The $\mathrm{Mg}$ contents increased significantly when treated with just boiled water bone + fish extracts compared to the control. In addition, the Mg contents were similar to the control when treated with fermentation tomato, boiled water red pepper leaf, and water bone + fish extracts. On the other hand, the Mg contents decreased significantly when treated with most of the other extracts compared to the control. Overall, most elements decreased significantly when treated with most extracts compared to the control.

Table 7. Nutrition levels of cucumber leaves treated with various extracts using drenching application at $1 \%$ concentrations.

\begin{tabular}{|c|c|c|c|c|c|c|}
\hline $\begin{array}{c}\text { Extraction } \\
\text { Method }\end{array}$ & Extract & T-N (\%) & $\mathrm{P}_{2} \mathrm{O}_{5}(\%)$ & $\mathrm{K}_{2} \mathrm{O}(\%)$ & $\mathrm{CaO}(\%)$ & $\operatorname{MgO}(\%)$ \\
\hline & Control & $4.05^{b c}$ & $0.73^{c}$ & $5.71^{a}$ & $2.41^{\mathrm{c}}$ & $1.21^{b c}$ \\
\hline \multirow{5}{*}{ Fermentation } & $\begin{array}{l}\text { Bone }+ \text { fish } \\
\text { meal }\end{array}$ & $3.63^{h *}$ & $0.59^{\mathrm{e}}$ & $5.10 \mathrm{def}$ & $2.10^{\mathrm{f}}$ & $1.09 \mathrm{fgh}$ \\
\hline & Tomato & $3.47^{\mathrm{i}}$ & $0.68^{\mathrm{d}}$ & $4.80^{\mathrm{h}}$ & $2.40^{\mathrm{c}}$ & $1.21^{\mathrm{b}}$ \\
\hline & Onion & $3.83^{\text {defg }}$ & $0.60^{\mathrm{e}}$ & $5.20^{\mathrm{cde}}$ & $2.08^{\mathrm{fg}}$ & $1.08 \mathrm{gh}$ \\
\hline & Red pepper leaf & 3.92 cde & $0.55^{f}$ & $5.06^{\text {efg }}$ & $2.05^{\mathrm{fgh}}$ & $1.12^{\text {defg }}$ \\
\hline & Sesame oil cake & $3.93 \mathrm{~cd}$ & $0.62^{e}$ & $5.71^{\mathrm{a}}$ & $2.28^{\mathrm{d}}$ & $1.13 \mathrm{de}$ \\
\hline \multirow{5}{*}{ Boiled water } & $\begin{array}{l}\text { Bone }+ \text { fish } \\
\quad \text { meal }\end{array}$ & $3.75 \mathrm{fgh}$ & $0.82^{b}$ & $5.28 \mathrm{bcd}$ & $2.59^{\mathrm{a}}$ & $1.28^{\mathrm{a}}$ \\
\hline & Tomato & $3.40^{\mathrm{i}}$ & $0.83^{b}$ & $4.89 \mathrm{gh}$ & $2.03 \mathrm{gh}$ & $1.05^{\mathrm{i}}$ \\
\hline & Onion & 3.77 efgh & $0.72 \mathrm{~cd}$ & $4.83^{\mathrm{h}}$ & $2.16^{\mathrm{e}}$ & $1.12 \mathrm{def}$ \\
\hline & Red pepper leaf & $4.10^{\mathrm{b}}$ & $0.91^{\mathrm{a}}$ & $5.40^{b c}$ & $2.25^{\mathrm{d}}$ & $1.18^{\mathrm{c}}$ \\
\hline & Sesame oil cake & 3.80 defg & $0.73^{c}$ & $4.90 \mathrm{fgh}$ & $2.24^{\mathrm{d}}$ & $1.14^{\mathrm{d}}$ \\
\hline \multirow{5}{*}{ Water } & $\begin{array}{c}\text { Bone }+ \text { fish } \\
\text { meal }\end{array}$ & $3.83^{\text {defg }}$ & $0.72^{\mathrm{cd}}$ & $5.69^{a}$ & $2.47^{\mathrm{b}}$ & $1.22^{b}$ \\
\hline & Tomato & $3.68 \mathrm{gh}$ & $0.74^{\mathrm{c}}$ & $5.43^{b}$ & $2.17^{\mathrm{e}}$ & $1.07^{\mathrm{h}}$ \\
\hline & Onion & 3.91 cdef & $0.72^{\mathrm{cd}}$ & $5.28 \mathrm{bcd}$ & $2.00^{\mathrm{h}}$ & $1.05^{\mathrm{i}}$ \\
\hline & Red pepper leaf & $4.40^{\mathrm{a}}$ & $0.72^{\mathrm{cd}}$ & $5.34^{b c}$ & $2.03 \mathrm{gh}$ & 1.11 efgh \\
\hline & Sesame oil cake & $4.03 \mathrm{bc}$ & $0.83^{b}$ & $5.78^{a}$ & $2.07 \mathrm{fg}$ & $1.10^{\mathrm{fgh}}$ \\
\hline
\end{tabular}

* Means within a column followed by the same letters are not significantly different at the $5 \%$ level according to Duncan's Multiple Range Test.

A previous study reported that the total $\mathrm{N}$ in the Peucedanum japonicum leaves increased significantly when treated with the Chinese chive extract at $0.5,1$, and $3 \%$ compared to the control [31]. On the other hand, the total $\mathrm{N}$ in Peucedanum japonicum leaves increased significantly when treated with tomato extracts only when the concentrations were higher than $3 \%$. In this study, extracts were used at only a $1 \%$ concentration. Thus, the levels of $\mathrm{N}$ and other elements can vary according to the treatment concentrations. Furthermore, if plants have larger biomass, they will need to absorb more nutrients from their treatments. These points may explain the decreased levels of elements in plants. Nevertheless, Crouch et al. [38] indicated that the use of seaweed concentrate led to significant increases in the $\mathrm{K}, \mathrm{Mg}$, and $\mathrm{Ca}$ concentrations in the leaves of lettuce plants that had received an adequate supply of nutrients. In a similar study, the $\mathrm{Cu}$ and Mo contents in the Peucedanum japonicum leaves increased significantly when treated with the soybean leaf, soybean stem, Chinese chive, and onion extracts compared to the control [31]. The extracts with the highest levels of growth-promoting characteristics did not necessarily produce plant leaves with the highest mineral nutrient contents. This means that growth promotion may not be caused by the high levels of a specific macro or micro element but rather by a specific combination of various elements. 


\subsection{Crude Protein, Crude Fat, and Ash Contents in Cucumber}

Cucumber fruit was harvested from the treated cucumber plants to determine crude protein, crude fat, and ash contents (Table 8). The fermentation red pepper leaf extract was the only extract that increased crude protein content in cucumber fruit significantly. Crude protein in the cucumber fruit from plants treated with other extracts was not significantly greater than the fruit from the control plants. The crude fat content in cucumber fruit was higher when treated with fermentation bone + fish, onion, and water tomato extracts than control and other extracts. On the other hand, the crude fat content was lower when treated with boiled water tomato, red pepper leaf, and sesame oil cake extracts than the control and other extracts. Fruit from the plants treated with other extracts had similar crude fat contents to the untreated control. The ash content in the cucumber fruit was higher when treated with the fermentation red pepper leaf and boiled water red pepper leaf extracts than the control or other extracts. In contrast, the ash content in plants treated with other extracts was similar to the untreated control. Overall, the levels of crude protein, crude fat, and ash content did not vary from the treated plants to the untreated control. In a previous study, the chemical composition of snap bean pods including organic nutrients, such as crude protein and total sugar, was enhanced significantly by applying a moringa leaf extract compared to the control [39]. On the other hand, the crude fibers were not enhanced significantly by the moringa leaf extract compared to the control. In another study, the sugar content in red pepper increased significantly when treated with a liquid fertilizer (fowl droppings: rice bran: soybean meal, 2:1:1) compared to the control [40].

Table 8. Crude protein, crude fat and ash contents in cucumber fruit from plants treated four times with various extracts at $1 \%$ concentrations.

\begin{tabular}{|c|c|c|c|c|}
\hline Extraction Method & Extract & Crude Protein (\%) & Crude Fat (\%) & Ash (\%) \\
\hline \multicolumn{2}{|r|}{ Control } & 10.995 & 0.74 & 11.33 \\
\hline \multicolumn{2}{|r|}{ Oil cake } & 11.62 & 0.68 & 10.90 \\
\hline \multirow{5}{*}{ Fermentation } & Bone + fish meal & 11.64 & 0.96 & 11.59 \\
\hline & Tomato & 10.35 & 0.75 & 10.60 \\
\hline & Onion & 11.42 & 0.98 & 10.46 \\
\hline & Red pepper leaf & 13.17 & 0.82 & 12.11 \\
\hline & Sesame oil cake & 11.02 & 0.77 & 10.67 \\
\hline \multirow{5}{*}{ Boiled water } & Bone + fish meal & 11.02 & 0.77 & 11.41 \\
\hline & Tomato & 10.89 & 0.49 & 11.17 \\
\hline & Onion & 10.25 & 0.64 & 10.79 \\
\hline & Red pepper leaf & 11.03 & 0.56 & 12.20 \\
\hline & Sesame oil cake & 11.98 & 0.59 & 10.86 \\
\hline \multirow{5}{*}{ Water } & Bone + fish meal & 11.24 & 0.89 & 10.56 \\
\hline & Tomato & 11.08 & 0.93 & 11.69 \\
\hline & Onion & 10.13 & 0.88 & 11.00 \\
\hline & Red pepper leaf & 10.84 & 0.74 & 10.04 \\
\hline & Sesame oil cake & 10.05 & 0.88 & 11.22 \\
\hline
\end{tabular}

\subsection{Phenol and Flavonoid Contents and DPPH Radical Scavenging Activity}

Many extracts, such as onion and tomato extracts, exhibit a range of bioactive properties and substances $[3,29,30]$. Thus, the extracts used in this study could affect phenol and flavonoid contents and DPPH radical scavenging activity. An ideal extract would have no adverse effects on the crop quality while also being an effective growth promoter. Therefore, the phenol and flavonoid contents and DPPH radical scavenging activity were measured in the cucumber fruit harvested from the treated plants (Table 9). Only plants treated with the water onion extract produced fruit with higher total phenol contents than the control. The total flavonoid contents in cucumber increased significantly when treated with the fermentation bone + fish extracts, but the plants treated with the other extracts had similar flavonoid contents to the fruit from control plants. DPPH radical scavenging 
activity in cucumber decreased significantly when treated with the oil cake and water bone + fish extracts compared to control. On the other hand, the fruit from plants treated with other extracts had similar DPPH radical scavenging activity to the fruit from control plants. Overall, most fruit from treated plants had similar total phenol and flavonoid contents and DPPH radical scavenging activity to the fruit taken from the control plants. In previous studies, total phenol and flavonoid contents and DPPH radical scavenging activity in the Peucedanum japonicum leaves treated with several extracts, such as Chinese chive and soybean leaf extracts at 0.5 to $3 \%$, increased by $23-81 \%$ compared to the control [40]. On the other hand, other extracts, such as onion and tomato extract-treated Peucedanum japonicum leaves, did not produce significantly different total phenol contents [31]. In another study, commercial Ascophyllum nodosum extracts improved the storage quality and flavonoid synthesis, and nutritional quality of the spinach leaf $[25,37]$.

Table 9. Total phenol, total flavonoid contents, and DPPH radical scavenging activity in cucumber fruit from plants treated four times with various extracts at $1 \%$ concentrations.

\begin{tabular}{|c|c|c|c|c|}
\hline Extraction Method & Extract & Phenol (mg/g) & Flavonoid (mg/g) & $\begin{array}{c}\text { DPPH Radical } \\
\text { Scavenging Activity } \\
(\%)\end{array}$ \\
\hline \multicolumn{2}{|c|}{ Control } & 4.08 bcde & 0.17 bcdef & $13.89 \mathrm{ab}$ \\
\hline \multicolumn{2}{|c|}{ Oil cake } & 3.43 de & $0.12^{\mathrm{f}}$ & $-9.61^{d}$ \\
\hline \multirow{6}{*}{ Fermentation } & Bone + fish meal & $4.96^{\mathrm{ab}}$ & $0.24^{\mathrm{a}}$ & $11.24^{b c}$ \\
\hline & Tomato & 4.21 abcde & $0.13^{\text {ef }}$ & $13.50^{\mathrm{ab}}$ \\
\hline & Onion & $4.41 \mathrm{abcd}$ & 0.17 bcdef & $15.28^{a}$ \\
\hline & Red pepper leaf & $3.11^{\mathrm{e}}$ & $0.15^{\text {cdef }}$ & $13.19^{a b}$ \\
\hline & Sesame oil cake & $4.43^{\mathrm{abcd}}$ & $0.18^{\text {abcdef }}$ & $12.95^{\mathrm{ab}}$ \\
\hline & Bone + fish meal & $4.37^{\mathrm{abcd}}$ & $0.21^{\mathrm{abc}}$ & $13.60^{\mathrm{ab}}$ \\
\hline \multirow{4}{*}{ Boiled water } & Tomato & $4.14^{\text {abcde }}$ & $0.13^{\text {def }}$ & $14.13^{\mathrm{ab}}$ \\
\hline & Onion & 3.85 bcde & $0.15^{\text {cdef }}$ & $12.30^{a b}$ \\
\hline & Red pepper leaf & 3.94 bcde & $0.15^{\text {cdef }}$ & $11.15^{b c}$ \\
\hline & Sesame oil cake & $4.62^{\mathrm{abc}}$ & $0.21^{\mathrm{abc}}$ & $11.39 \mathrm{bc}$ \\
\hline \multirow{5}{*}{ Water } & Bone + fish meal & $4.75^{\mathrm{abc}}$ & $0.23^{\mathrm{ab}}$ & $8.84^{\mathrm{c}}$ \\
\hline & Tomato & 3.69 cde & $0.13^{\text {def }}$ & $14.75^{\mathrm{a}}$ \\
\hline & Onion & $5.22^{\mathrm{a}}$ & $0.20^{\mathrm{abcd}}$ & $12.68^{\mathrm{ab}}$ \\
\hline & Red pepper leaf & 4.09 abcde & 0.19 abcde & $15.23^{a}$ \\
\hline & Sesame oil cake & $4.71^{\mathrm{abc}}$ & $0.19^{\text {abcde }}$ & $14.51^{\mathrm{a}}$ \\
\hline
\end{tabular}

* Means within a column followed by the same letters are not significantly different at the $5 \%$ level according to Duncan's Multiple Range Test.

These studies measured total phenol, total flavonoid contents, and DPPH radical scavenging activity in the leaf-treated extracts. On the other hand, total phenol, total flavonoid contents, and DPPH radical scavenging activity in harvested cucumber fruit were measured after the extract treatments in the leaves. Fortunately, the growth-promoting extracts used in this study did not adversely affect the secondary metabolites.

\section{Conclusions}

Over the course of this study, we determined that some extracts are suitable for use in organic cucumber crop cultivation. Compared to the control, cucumber fruit weight was $17-81 \%, 10-61 \%$, and $10-45 \%$ higher after receiving applications of fermentation, boiled water, and water extracts, respectively. Generally, cucumber plant height also increased both 7 and 14 days after treatments regardless of which extraction method or extraction materials were used to produce the extracts. The application frequency effects differed depending on extraction method and extract material. Combinations of extracts did not produce greater growth promotion than the extracts would have produced individually. Generally, crude protein, crude fat, ash, phenol, flavonoid contents, and DPPH radical scavenging activity were not adversely affected by the extract treatments. However, addi- 
tional studies could find liquid fertilizers that increase primary and secondary substances. Despite the growth-promoting effects of some of the tested fertilizers, chlorophyll content and photosynthetic efficiency of the cucumber plants did not vary significantly regardless of which application plants received. Therefore, more studies are needed in order to better understand the mechanisms of growth promotion.

Author Contributions: Data curation, H.H.P.; writing - original draft preparation, S.J.J.; writingreview and editing, Y.I.K. All authors have read and agreed to the published version of the manuscript.

Funding: This work was carried out with the support of the "Cooperative Research Program for Agriculture Science \& Technology Development (Project No. PJ01338805)" Rural Development Administration, Republic of Korea.

Institutional Review Board Statement: Not applicable.

Informed Consent Statement: Not applicable.

Data Availability Statement: Not applicable.

Acknowledgments: The authors acknowledge the help of Min Hee Park, Hyo Jin Lee, and Ok Gi Lee in crop cultivation.

Conflicts of Interest: The authors declare no conflict of interest.

\section{References}

1. Chae, J.C.; Gang, B.H.; Park, S.J.; Kim, S.H. Samgo Cultivation Principles; Hyangmunsa: Seoul, Korea, 2008; p. 434. (In Korean)

2. An, N.H.; Kim, Y.K.; Lee, Y.; Jee, H.J.; Park, J.H.; Hong, S.J.; Han, E.J. Changes in chemical properties and microbial population of farm-made organic liquid fertilizer during fermenting process. Korean J. Org. Agric. 2011, 19, 417-425.

3. Jang, S.J.; Kuk, Y.I. Crop growth promotion and mineral nutrient levels in various extracts of onion (Allium cepa) and tomato (Lycopersicon esculentum). Res. Crops 2020, 21, 315-326.

4. Hartz, T.K.; Smith, R.; Gaskell, M. Nitrogen availability from liquid organic fertilizers. HortTechnology 2010, 20, 169-172. [CrossRef]

5. Kauffman, G.L.; Kneivel, D.P.; Watschke, T.L. Effects of a biostimulant on the heat tolerance associated with photosynthetic capacity, membrane thermostability, and polyphenol production of perennial ryegrass. Crop. Sci. 2007, 47, 261-267. [CrossRef]

6. Kuk, Y.I.; Yun, Y.B.; Jang, S.J.; Jeong, J.Y.; Kim, D.S.; Kim, S.S. Evaluation of tomato growth-promoting effect and mineral nutrient of farm-made liquid fertilizers. Korean J. Org. Agric. 2019, 27, 205-224.

7. Jang, S.J.; Kuk, Y.I. Evaluation on the performance of red pepper (Capsicum annuum) and tomato (Lycopersicon esculentum) under various fermented liquid fertilizers. Res. Crops 2020, 21, 306-314.

8. Liedl, B.E.; Bombardiere, J.; Chaffield, J.M. Fertilizer potential of liquid and solid effluent from thermophilic anaerobic digestion of poultry waste. Water Sci. Technol. 2006, 53, 69-79. [CrossRef]

9. Zhai, Z.; Ehret, D.L.; Forqe, T.; Helmer, T.; Lin, W.; Dorais, M.; Papadopoulos, A.P. Organic fertilizers for greenhouse tomatoes: Productivity and microbiology. HortScience 2009, 44, 800-809. [CrossRef]

10. An, N.H.; Jo, Y.S.; Jo, J.R.; Kim, Y.K.; Lee, Y.; Jee, H.J.; Lee, S.M.; Park, K.L.; Lee, B.M. The survey of actual using conditions of farm-made liquid fertilizers for cultivating environment-friendly agricultural products. Korean J. Org. Agric. 2012, 20, 345-356.

11. Khan, W.; Rayirath, U.P.; Subramanian, S.; Jithesh, M.N.; Rayorath, P.; Hodges, D.M.; Critchley, A.T.; Craigie, J.S.; Norrie, J.; Prithiviraj, B. Seaweed extracts as biostimulants of plant growth and development. J. Plant. Growth Regul. 2009, $28,386-399$. [CrossRef]

12. Jannin, L.; Arkoun, M.; Etienne, P.; Laîné, P.; Goux, D.; Garnica, M.; Fuentes, M.; Francisco, S.S.; Baigorri, R.; Cruz, F. Brassica napus growth is promoted by Ascophyllum nodosum (L.) Le Jol. seaweed extract: Microarray analysis and physiological characterization of N, C, and S metabolisms. J. Plant. Growth Regul. 2013, 32, 31-52. [CrossRef]

13. Mancuso, S.; Azzarello, E.; Mugnai, S.; Briand, X. Marine bioactive substance (IPA extract) improve foliar ion uptake and water stress tolerance in potted Vitis vinifera plants. Adv. Hortic. Sci. 2006, 20, 156-161.

14. Spinelli, F.; Fiori, G.; Noferini, M.; Sprocatti, M.; Costa, G. Perspectives on the use of a seaweed extract to moderate the negative effects of alternate bearing in apple trees. J. Hortic. Sci. Biotechnol. 2009, 84, 131-137. [CrossRef]

15. Bulgari, R.; Cocetta, G.; Trivellini, A.; Vernieri, P.; Ferrante, A. Biostimulants and crop responses: A review. Biol. Agric. Hortic. 2015, 31, 1-17. [CrossRef]

16. El Sohaimy, S.A.; Hamad, G.M.; Mohamed, S.E.; Amar, M.H.; Al-Hindi, R.R. Biochemical and functional properties of Moringa oleifera leaves and their potential as a functional food. Global Adv. Res. J. Agric. Sci. 2015, 4, 188-199.

17. Yasmeen, A.; Basra, S.M.A.; Farooq, M.; Rehman, H.; Hussain, N.; Athar, H.R. Exogenous application of moringa leaf extract modulates the antioxidant enzyme system to improve wheat performance under saline conditions. Plant. Growth Regul. 2013, 69, 225-233. [CrossRef] 
18. Phiri, C. Influence of Moringa oleifera leaf extracts on germination and early seedling development of major cereals. Agric. Biol. J. N. Am. 2010, 1, 774-777. [CrossRef]

19. Fernie, A.R.; Pichersky, E. Focus issue on metabolism: Metabolites, metabolites everywhere. Plant. Physiol. 2015, 169, 1421-1423. [CrossRef]

20. Liu, J.; Osbourn, A.; Ma, P. MYB transcription factors as regulators of phenylpropanoid metabolism in plants. Mol. Plant. 2015, 8, 689-708. [CrossRef] [PubMed]

21. Sarker, U.; Oba, S. Drought stress enhances nutritional and bioactive compounds, phenolic acids and antioxidant capacity of Amaranthus leafy vegetable. BMC Plant. Biol. 2018, 18, 1-15. [CrossRef]

22. Sarker, U.; Oba, S. Salinity stress enhances color parameters, bioactive leaf pigments, vitamins, polyphenols, flavonoids and antioxidant activity in selected Amaranthus leafy vegetables. J. Sci. Food Agric. 2019, 99, 2275-2284. [CrossRef] [PubMed]

23. Sarker, U.; Oba, S. The response of salinity stress induced A. tricolor to growth, anatomy, physiology, non-enzymatic and enzymatic antioxidants. Front. Plant. Sci. 2020, 11, 559876. [CrossRef]

24. Sarker, U.; Oba, S. Drought stress effects on growth, ROS markers, compatible, phenoic, flavonoids, and antioxidant activity in Amaranthus tricolor. Appl. Biotechnol. 2018, 186, 999-1016.

25. Fan, D.; Hodges, D.M.; Zhang, J.; Kirby, C.W.; Ji, X.; Locke, S.J.; Critchley, A.T.; Prithiviraj, B. Commercial extract of the brown seaweed Ascophyllum nodosum enhances phenolic antioxidant content of spinach (Spinacia oleracea L.) which protects Caenorhabditis elegans against oxidative and thermal stress. Food Chem. 2011, 124, 195-202. [CrossRef]

26. Jang, S.J.; Kuk, Y.I. Growth promotion of cucumber (Cucumis sativus) plants using various extracts with different extraction and application methods. Res. Crops 2020, 21, 756-768.

27. RDA. Methods of Soil and Plant Analysis. Rural Development Administration; Sammi Press: Suwon, Korea, $2000 ;$ pp. 1-202.

28. AOAC. Official Methods of Analysis, 18th ed.; Association of Officiating Analytical Chemists: Washington, DC, USA, 2005.

29. Jang, S.J. Control of Diseases, Insects, and Weeds and Growth Promotion of Crops by Useful Plant Extracts. Ph.D. Dissertation, South Korea National University, Seoul, Korea, 2018; p. 205.

30. Jang, S.J.; Kuk, Y.I. Growth promotion effects of plant extracts on various leafy vegetable crops. Hortic. Sci. Technol. 2019, 37, 322-336.

31. Jang, S.J.; Park, H.H.; Kuk, Y.I. Growth promotion, nutrition levels, and antioxidant activity in Peucedanum japonicum Thunb. under various plant extracts. Agronomy 2020, 10, 1494. [CrossRef]

32. SAS (Statistical Analysis System). SAS/STAT User's Guide, Version 7; Statistical Analysis System Institute, Electronic Version: Cary, NC, USA, 2000.

33. Khan, S.; Basra, S.M.A.; Nawaz, M.; Hussain, I.; Foidl, N. Combined application of moringa leaf extract and chemical growthpromoters enhances the plant growth and productivity of wheat crop (Triticum aestivum L.). S. Afr. J. Bot. 2020, $129,74-81$. [CrossRef]

34. Pereira, L.D.M.; Pereira, E.D.M.; Revolti, L.T.M.; Zingaretti, S.M.; Môro, G.V. Seed quality, chlorophyll content index and leaf nitrogen levels in maize inoculated with Azospirillum brasilense. Rev. Ciênc. Agron. 2015, 46, 630-637. [CrossRef]

35. Latif, H.; Mohamed, H. Exogenous applications of moringa leaf extract effect on retrotransposon, ultrastructural and biochemical contents of common bean plants under environmental stresses. S. Afr. J. Bot. 2016, 106, 221-231. [CrossRef]

36. Fan, D.; Hodges, D.M.; Critchley, A.T.; Prithiviraj, B. A commercial extract of brown macroalga (Ascophyllum nodosum) affects yield and the nutritional quality of spinach in vitro. Commun. Soil Sci. Plant. Anal. 2013, 44, 1873-1884. [CrossRef]

37. Battacharyya, D.; Babgohari, M.Z.; Rathor, P.; Prithiviraj, B. Seaweed extracts as biostimulants in horticulture. Sci. Hortic. 2015, 196, 39-48. [CrossRef]

38. Crouch, I.J.; Beckett, R.; Van Staden, J. Effect of seaweed concentrate on the growth and mineral nutrition of nutrient stressed lettuce. J. Appl. Phycol. 1990, 2, 269-272. [CrossRef]

39. Elzaawely, A.A.; Ahmed, M.E.; Maswada, H.F.; Xuan, T.D. Enhancing growth, yield, biochemical, and hormonal contents of snap bean (Phaseolus vulgaris L.) sprayed with moringa leaf extract. Arch. Agron. Soil Sci. 2017, 63, 687-699. [CrossRef]

40. Joo, S.J.; Lee, G.J. Effects of organic liquid fertilizer fertigation on growth and fruit quality of hot pepper. Korean J. Org. Agric. 2010, 18, 63-74. 